\title{
Hippocampal Respiration-Driven Rhythm Distinct from Theta Oscillations in Awake Mice
}

\author{
Vivan Nguyen Chi, ${ }^{1}$ Carola Müller, ${ }^{1}$ Thérèse Wolfenstetter, ${ }^{1}$ Yevgenij Yanovsky, ${ }^{1}$ Andreas Draguhn, ${ }^{1}$ \\ DAdriano B.L. Tort, ${ }^{2 \star}$ and Jurij Brankačk ${ }^{1 \star}$ \\ ${ }^{1}$ Institute for Physiology and Pathophysiology, University Heidelberg, 69120 Heidelberg, Germany, and ²Brain Institute, Federal University of Rio Grande \\ do Norte, 59056-450 Natal, Rio Grande do Norte, Brazil
}

We have recently described a slow oscillation in the hippocampus of urethane-anesthetized mice, which couples to nasal respiration and is clearly distinct from co-occurring theta oscillations. Here we set out to investigate whether such type of patterned network activity, which we named "hippocampal respiration rhythm" (HRR), also occurs in awake mice. In freely moving mice, instantaneous respiration rate is extremely variable, and respiration is superimposed by bouts of sniffing. To reduce this variability, we clamped the behavior of the animal to either awake immobility or treadmill running by using a head-fixed setup while simultaneously recording respiration and field potentials from the olfactory bulb $(\mathrm{OB})$ and hippocampus. Head-fixed animals often exhibited long periods of steady respiration rate during either immobility or running, which allowed for spectral and coherence analyses with a sufficient frequency resolution to sort apart respiration and theta activities. We could thus demonstrate the existence of HRR in awake animals, namely, a respiration-entrained slow rhythm with highest amplitude at the dentate gyrus. HRR was most prominent during immobility and running with respiration rates slower than theta oscillations. Nevertheless, HRR could also be faster than theta. Discharges of juxtacellularly recorded cells in CA1 and dentate gyrus were modulated by HRR and theta oscillations. Granger directionality analysis revealed that HRR is caused by the OB and that theta oscillations in $\mathrm{OB}$ are caused by the hippocampus. Our results suggest that respiration-coupled oscillations aid the exchange of information between olfactory and memory networks.

Key words: hippocampus; mouse; olfactory bulb; oscillation; respiration; theta rhythm

\section{Significance Statement}

Olfaction is a major sense in rodents. In consequence, the olfactory bulb $(\mathrm{OB})$ should be able to transmit information to downstream regions. Here we report potential mechanisms underlying such information transfer. We demonstrate the existence of a respiration-entrained rhythm in the hippocampus of awake mice. Frequencies of the hippocampal respiration rhythm (HRR) overlap with classical theta oscillations, but both rhythms are clearly distinct. HRR is most prominent in the dentate gyrus, especially when respiration is slower than theta frequency. Discharges of neurons in CA1 and dentate gyrus are modulated by both HRR and theta. Directionality analysis shows that HRR is caused by the OB. Our results suggest that respiration-coupled oscillations aid the exchange of information between olfactory and memory networks.

\section{Introduction}

The brain exhibits a variety of rhythms that have been linked to different structures, behaviors, and cognitive states. It is currently

Received July 29, 2015; revised Nov. 10, 2015; accepted Nov. 17, 2015.

Author contributions: Y.Y., A.D., A.B.L.T., and J.B. designed research; V.N.C., C.M., T.W., and Y.Y. performed research; A.B.L.T. and J.B. analyzed data; Y.Y., A.D., A.B.L.T., and J.B. wrote the paper.

This work was supported by the Deutsche Forschungsgemeinschaft (SFB 636/B06), Bundesministerium für Bildung und Forschung (Bernstein Center for Computational Neurosciences 01GQ1003A; German-Brazil Cooperation Grant 01DN12098), and the Brazilian National Council for Scientific and Technological Development (CNPq).

The authors declare no competing financial interests.

${ }^{*}$ A.B.L.T. and J.B. contributed equally to this work.

Correspondence should be addressed to Dr. Jurij Brankačk, Institute for Physiology, and Pathophysiology, University Heidelberg, Im Neuenheimer Feld 326, 69120 Heidelberg, Germany. E-mail: jurij.brankack@physiologie.uni-heidelberg.de. thought that brain rhythms play functional roles in network computation, such as in mnemonic processes and decisionmaking (Miltner et al., 1999; Montgomery and Buzsáki, 2007; Buzsáki and Moser, 2013). Specifically, subthreshold oscillations provide a common synchronizing signal throughout functional networks, enabling temporally coordinated multineuronal activity patterns (Buzsáki and Draguhn, 2004). Spatially extended network oscillations may aid long-range communication by defining synchronous time windows for information exchange between remote groups of neurons (Buzsáki, 2010). 
The hippocampus of rodents expresses prominent theta oscillations during active behaviors and REM sleep (Vanderwolf, 1969; Buzsáki, 2002). Hippocampal theta is thought to constitute a synchronizing signal for various neocortical and subcortical regions (Sirota et al., 2008; Battaglia et al., 2011; Igarashi et al., 2013). However, the hippocampus receives multimodal sensory information through the entorhinal cortex (Brankačk and Buzsáki, 1986), and hippocampal oscillations may also reflect neocortical inputs to this region (Colgin et al., 2009; Schomburg et al., 2014).

Olfaction is a dominant sense in rodents, as reflected in their relatively large olfactory bulb $(\mathrm{OB})$. As in other brain regions, oscillations also hallmark network activity in OB (Adrian, 1942; Vanderwolf, 1992; Fontanini and Bower, 2006; Kay et al., 2009). Local field potentials (LFPs) from the OB exhibit a large amplitude rhythm entrained by respiration; that is, the instantaneous frequency of this rhythm, here called respiration rhythm (RR), exactly matches breathing rate (Klingberg et al., 1964; Vanderwolf and Szechtman, 1987). Fontanini et al. (2003) demonstrated that, under ketaminexylazine anesthesia, RR in rats reaches the piriform cortex, where it can be detected both extracellularly and intracellularly, and proposed that RR plays a chief role in the communication between $\mathrm{OB}$ and piriform cortex.

More recently, we have shown that RR actually reaches a further downstream region in urethane-anesthetized mice, namely, the hippocampus (Yanovsky et al., 2014), where the rhythm has been dubbed hippocampal respiration rhythm (HRR). Although a clearly distinct network pattern, HRR can occur simultaneously with hippocampal theta oscillations and, importantly, HRR and theta can have overlapping peak frequencies depending on respiration rate. Among other implications, this finding raises the possibility that previous work on hippocampal theta oscillations could actually have been "contaminated" by HRR activity.

The long-range effect of OB RR described above, however, was found during anesthesia (Fontanini et al., 2003; Yanovsky et al., 2014), which renders artificial brain states. Here we investigate how respiration-related rhythms and theta oscillations interact in awake, behaving animals during natural brain states. To that end, we measured respiration and field potential oscillations during rest and locomotion of awake head-fixed mice. We found mutual, statedependent interactions between hippocampal and olfactory networks in both patterns: theta and respiration-coupled oscillations. Specifically, our results show that respiration is an important synchronizing signal in the rodent brain, reaching far beyond classical olfactory networks.

\section{Materials and Methods}

In short, we recorded LFPs from the hippocampus of awake mice with simultaneous measurement of respiratory activity using thermocouples. Thirty-five animals were recorded in head-fixed conditions. Thermocouples were chronically implanted into the nasal cavity or placed in front of the nostrils. In 12 animals, we implanted NeuroNexus silicon probes with 16 recording sites separated by $100 \mu \mathrm{m}$ across the CA1dentate gyrus (DG) axis. Anatomical landmarks were identified by stimulation of the angular bundle (Brankačk et al., 1993; Yanovsky et al., 2014). Recording sites started above stratum oriens and reached to the ventral blade of the DG. In all mice, simultaneous LFP recordings were obtained from OB. Below we provide further methodological details.

Ethics statement. This study was performed in accordance with guidelines of the European Science Foundation (2001), the National Institutes of Health (National Research Council, 1996), and has been approved by the Governmental Supervisory Panel on Animal Experiments of Baden Württemberg at Karlsruhe (35-9185.81/G-84/13). All efforts were made to minimize animal suffering and to reduce the number of animals used.
Because of the systemic approach of the study, alternatives to in vivo techniques were not available.

Animal care and housing conditions. C57BL/6N mice were purchased at 70 or $84 \mathrm{~d}$ of age from Charles River. Animals were housed in groups of 4 inside a ventilated Scantainer (Scanbur BK) on an inverted 12/12 h light/ dark cycle (light on 8:00 P.M.) for a minimum of 2 weeks. Animals had free access to water and food. After chronic electrode implantation, mice were housed individually throughout the experiment. The animals were killed with an overdose of isoflurane during brain perfusion.

Animal preparation. A total of 35 (29 female and 6 male) C57BL/6N mice weighting $18-32 \mathrm{~g}$ (12-20 weeks old) were anesthetized with isoflurane in medical oxygen ( $4 \%$ isoflurane for induction, $1.5 \%-2.5 \%$ for maintenance, flow rate: 11 per minute). For analgesia, $0.1 \mathrm{mg} / \mathrm{kg}$ of buprenorphine was injected subcutaneously before and $8 \mathrm{~h}$ after surgery. Anesthetized animals were mounted on a stereotaxic apparatus (Kopf Instruments) with a custom-made inhalation tube. Body temperature was maintained at $38^{\circ} \mathrm{C}$ by a heating pad (ATC-2000, World Precision Instruments). After exposure of the skull, holes of $0.5-1.0 \mathrm{~mm}$ in diameter were drilled above the dorsal hippocampus and $\mathrm{OB}$ according to stereotaxic coordinates (see below for coordinates based on Paxinos and Franklin, 2001). Two stainless steel watch screws $(1 \times 3 \mathrm{~mm})$ over the cerebellum served as ground and reference electrode. For monitoring the temperature of nasal air flow, a precision fine bare wire thermocouple (80 $\mu \mathrm{m}$ diameter, Omega Engineering, part \#5TC-TT-KI-40-1M) was implanted into the right nasal cavity $[11 \mathrm{~mm}$ anteroposterior (AP), $0.5 \mathrm{~mm}$ mediolateral (ML)]. Two pairs of varnish-insulated tungsten wires (50 $\mu \mathrm{m}$, glued together) cut at an angle of $45^{\circ}$ were implanted into the right dorsal hippocampal CA1/DG area [-2.0 mm AP, $1.5 \mathrm{~mm} \mathrm{ML}, 1.5-2.5$ $\mathrm{mm}$ dorsoventral (DV)]. A pair of $125 \mu \mathrm{m}$ nichrome electrodes was positioned at the angular bundle $(-4.2 \mathrm{~mm} \mathrm{AP}, 2.75 \mathrm{~mm} \mathrm{ML}, 1.8 \mathrm{~mm}$ DV) for perforant path stimulation $(60-350 \mu \mathrm{A})$. In 2 animals, an additional pair of nichrome electrodes was placed at the lateral olfactory tract (LOT, 4.0 mm AP, $0.8 \mathrm{~mm}$ ML, $2.5 \mathrm{~mm} \mathrm{DV}$ ) for stimulation (200-450 $\mu \mathrm{A})$. For restraining the head of the animal in head-fixed experiments, a steel pin ( $2.1 \mathrm{~mm}$ diameter, $14 \mathrm{~mm}$ length) was attached to the skull under an angle of 75 degrees. Silicon probes (A1x16-3 mm-100-703CM16LP, NeuroNexus Technologies) were chronically implanted into the dorsal hippocampus in 10 animals $(-2.0 \mathrm{~mm}$ AP, $1.5 \mathrm{~mm} \mathrm{ML}, 2.2-$ $2.5 \mathrm{~mm}$ DV for the deepest position).

For juxtacellular recordings, a $0.5 \mathrm{~mm}$ hole was drilled into the skull above the right dorsal hippocampus ( $-2.0 \mathrm{~mm} \mathrm{AP}, 1.8 \mathrm{~mm} \mathrm{ML}$ ). A plastic ring (2 $\mathrm{mm}$ diameter, $1.5 \mathrm{~mm}$ high) was fixed above the hole and filled with paraffin oil (AppliChem) to prevent the brain surface from drying up. Between experiments, the plastic ring was covered with bone wax. Reference and ground electrodes consisted of chlorided silver wire with a diameter of 0.5 $\mathrm{mm}$ inserted into $0.6 \mathrm{~mm}$ holes above the cerebellum and fixed with flowable restorative composite (Filtek SupremeXTE, 3M ESPE). Two additional electrodes for LFP recordings were placed at the surface of the right $\mathrm{OB}(4.2 \mathrm{~mm}$ $\mathrm{AP}, 0.5 \mathrm{~mm} \mathrm{ML}$ ) and at the left dorsal hippocampus (-2.0 $\mathrm{mm} \mathrm{AP}, 1.5 \mathrm{~mm}$ ML, $2.0 \mathrm{~mm} \mathrm{DV}$ ) as described above.

Electrophysiology. One week after surgery, experiments began with a $1 \mathrm{~h}$ session in the animal's home cage or in a custom-built head-fixed setup.

Head-fixed recordings in awake mice. Figure $1 C$ shows a scheme of the head restraining device, a modified stereotaxic apparatus. The animal's head was fixed by the attached head pin to the stereotaxic positioner. The mouse was able to run on a circular treadmill made of autoclavable polycarbonate (UNO BV). A sensor attached to the circular treadmill recorded the running speed. All mice habituated quickly to the head restraining conditions and tolerated it well for up to $1 \mathrm{~h}$. The behavior of the head-restrained animal was mainly restricted to immobility and running. Hence, the respiration rate in head-fixed experiments was much more stable compared with freely moving conditions (compare Figs. 1D,E). Most animals ran voluntarily. Alternatively, a motor could be attached to the treadmill to enforce running at a stable speed. However, enforced running was mainly used to teach animals to run voluntarily; for each animal, we used data from voluntary running whenever possible: from the 21 animals recorded during running, 19 ran voluntarily and 2 were forced to run. Extracellular signals were filtered $(1-500 \mathrm{~Hz})$, ampli- 
A

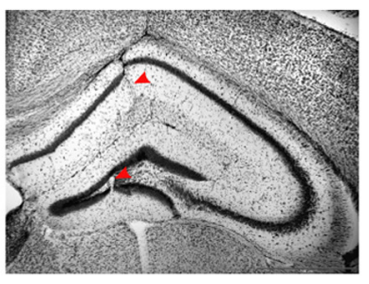

B

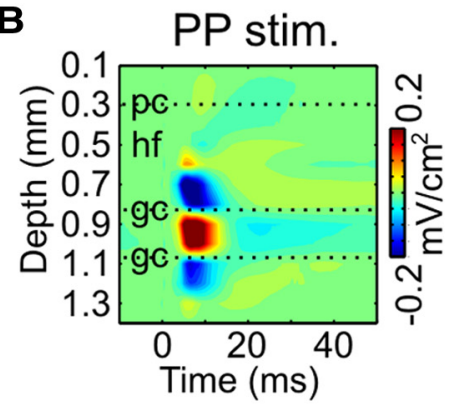

D

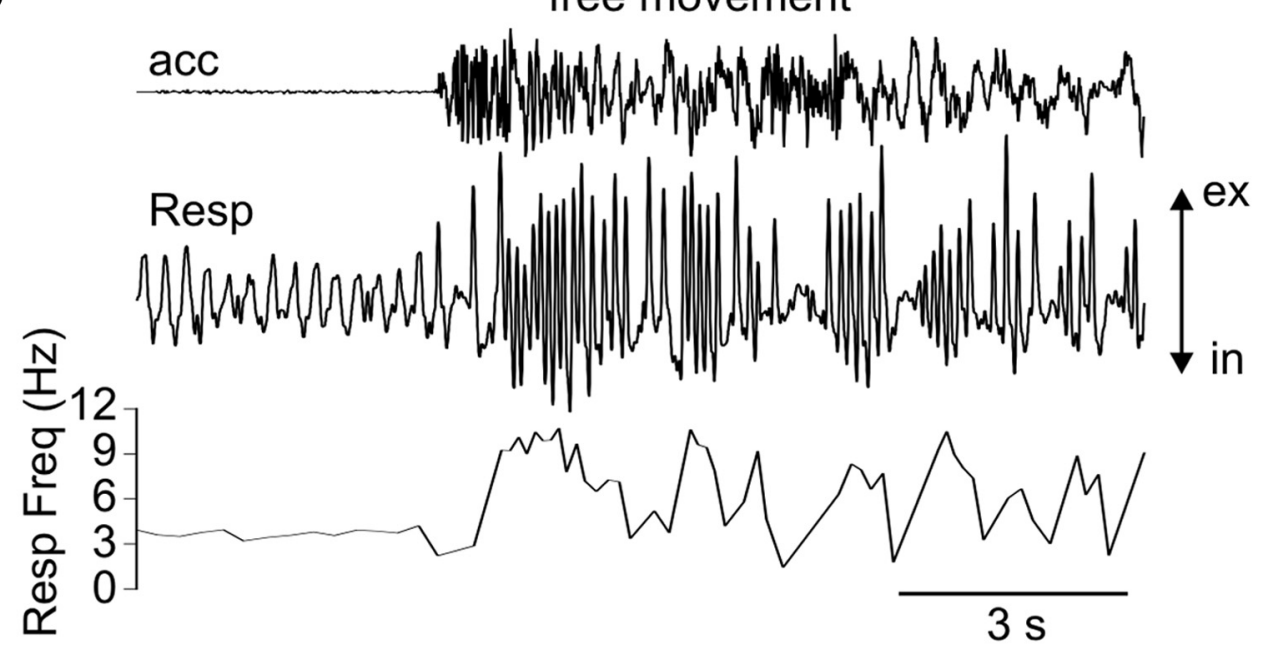

$\mathbf{E}$ acc head fixed

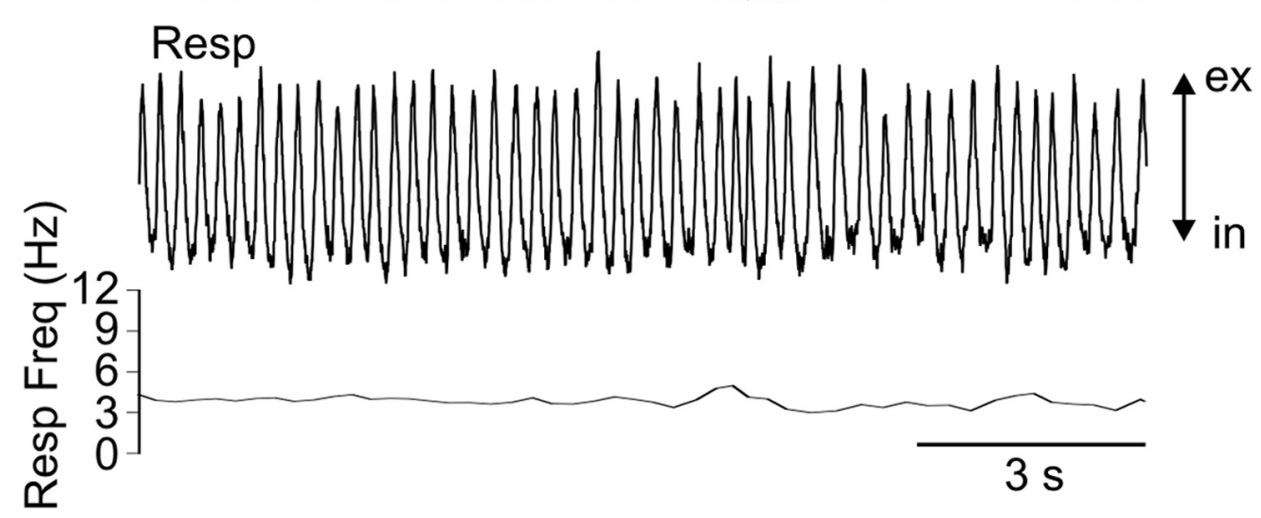

Figure 1. Simultaneous recording of motion, nasal respiration, and LFPs from the hippocampus and OB in awake mice. $A$, Localization of a multichannel probe inserted across the CA1-DG axis verified by histology (red arrowheads). $B$, Current source density analysis of LFPs in response to stimulation of perforant pathway (PP stim) used to identify anatomical landmarks. pc, Pyramidal cell layer; hf, hippocampal fissure; gc, granular cell layer. C, Setup for head-fixed experiments over a circular treadmill. An external thermocouple in front of the animal's nose records respiration. Speed is recorded during voluntary or forced running. Hip, Hippocampus; OB, olfactory bulb. D, Recordings of motion (acc: accelerometer), nasal respiration (Resp), and instantaneous frequency of respiration (Resp Freq) reveal high variability of respiration rate during free movement in the home cage. $\boldsymbol{E}$, Stationary respiration rate during head-fixed experiments. ex, Expiration; in, inspiration. 

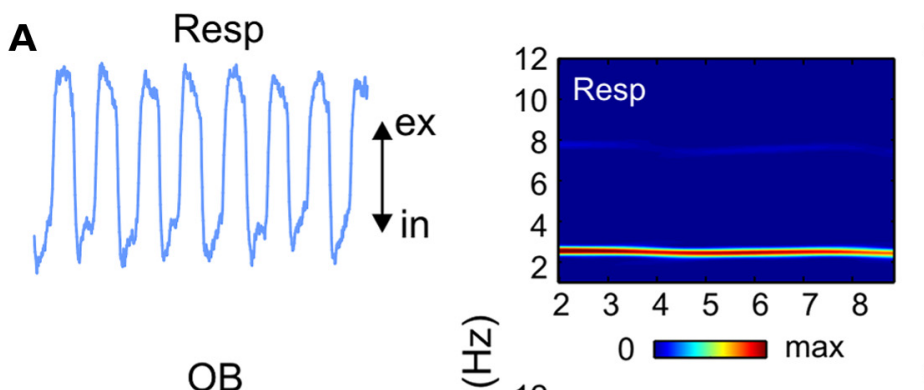

B
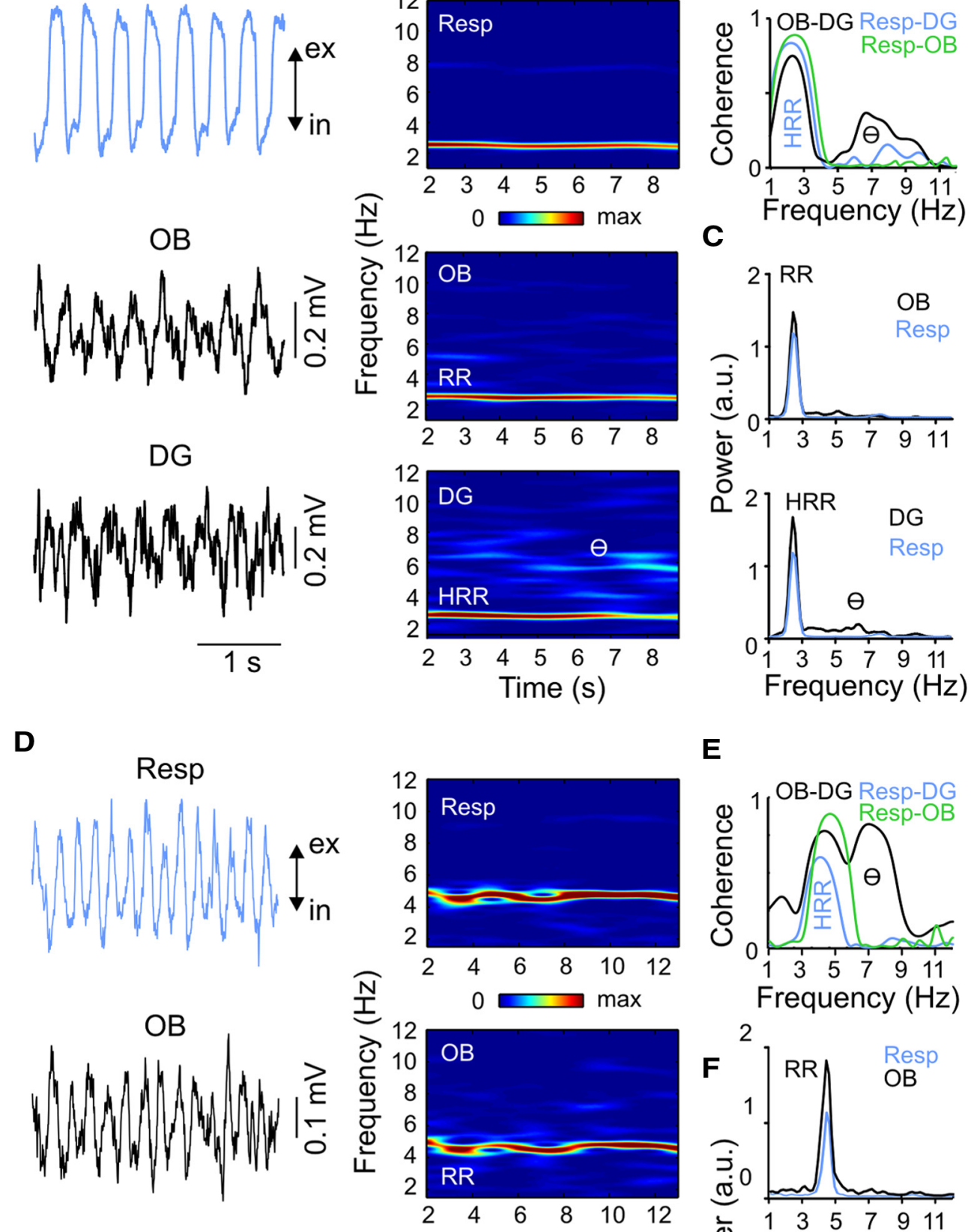

E
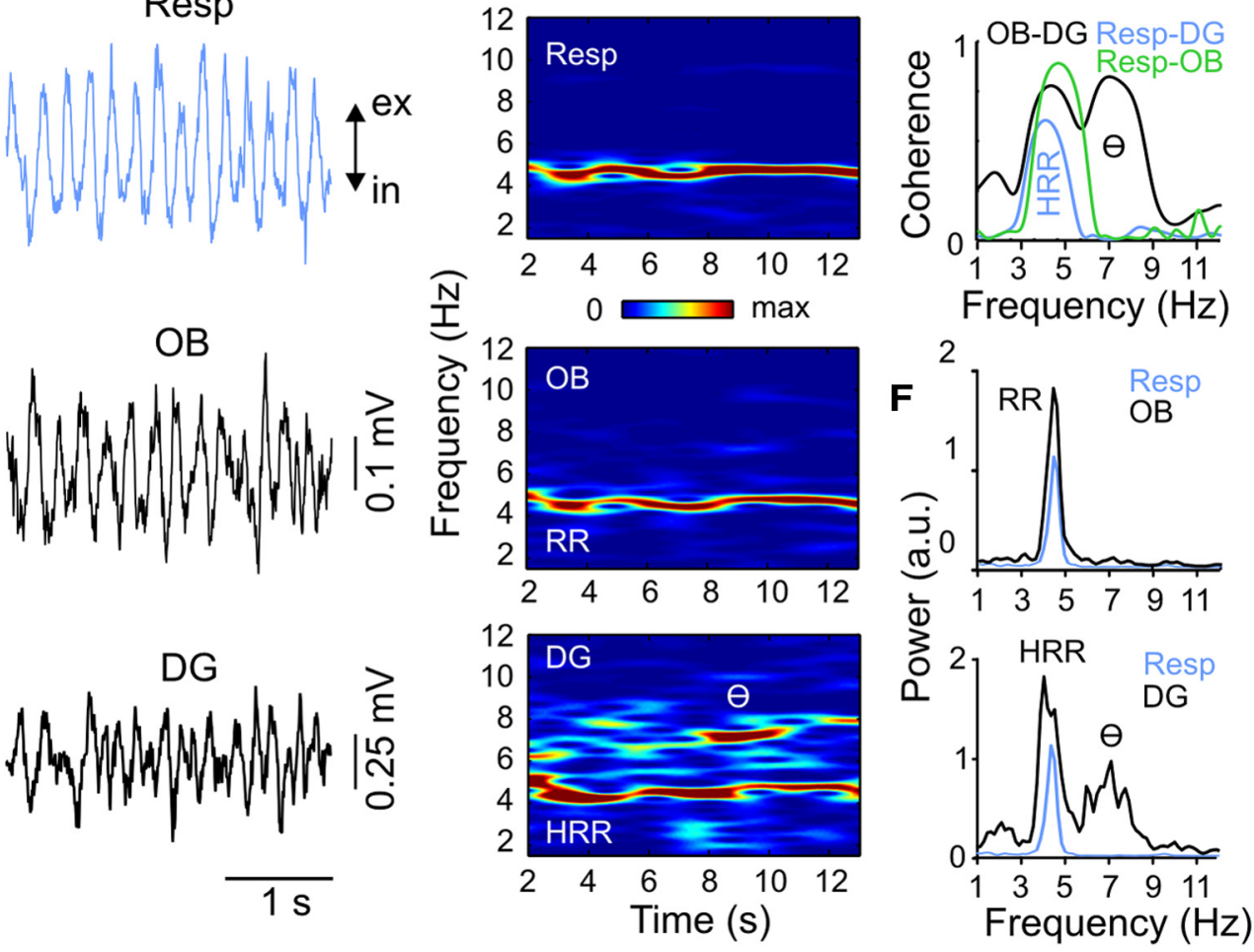

Figure 2. Respiration-entrained rhythm in hippocampus and $O B$ during immobility. $A, D$, Raw signals (left) and time-frequency power distributions (right) of respiration (Resp, blue line) and LFPs (black lines) in $O B$ and $D G$ during immobility with weak $(\boldsymbol{A})$ and strong $(\boldsymbol{D})$ theta oscillations $(\theta)$ in two representative animals. Notice, in both examples, prominent respiration-entrained rhythms in $O B$ and DG LFPs, which are referred to as RR and HRR. ex, Expiration; in, inspiration. $B, E$, Coherence spectra between $O B$ and DG LFPs (black), between Resp and DG LFP (blue), and between Resp and OB LFP (green). Notice high coherence at respiration frequency for all pairs. In addition, coherence between $0 B$ and DG also peaks at theta frequency. C, $F$, Power spectra for OB and DG LFPs (black) plotted along with respiration power (blue). 
A

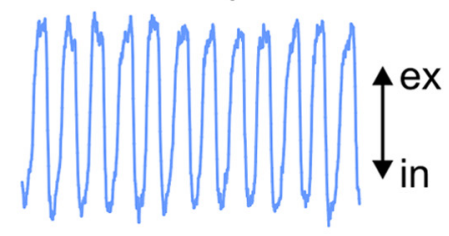

Resp

OB

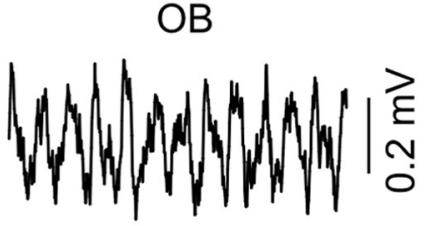

DG

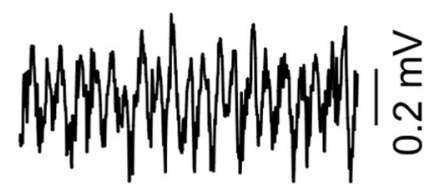

$1 \mathrm{~s}$

\section{กิ}

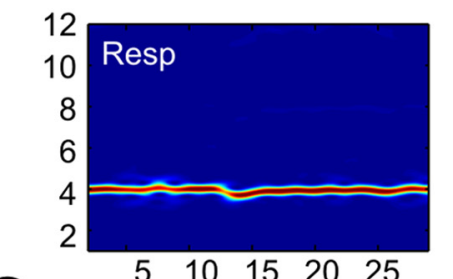

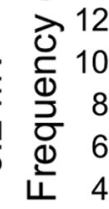
2
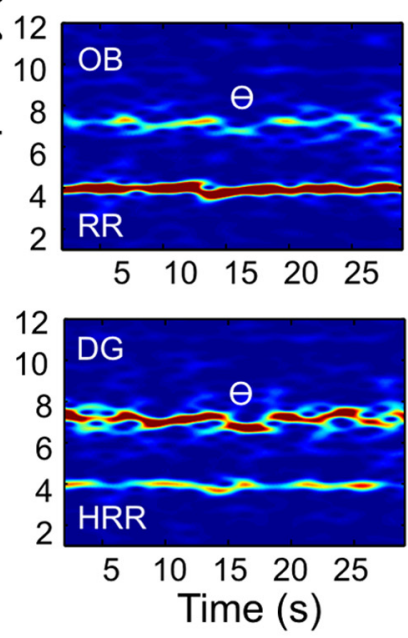

B

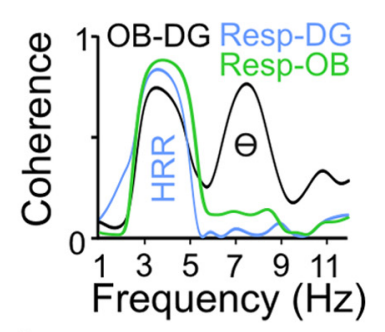

C

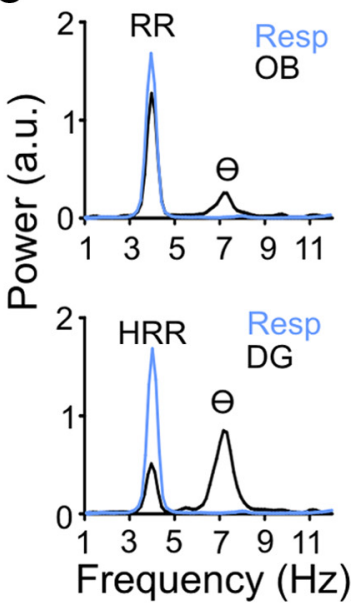

E
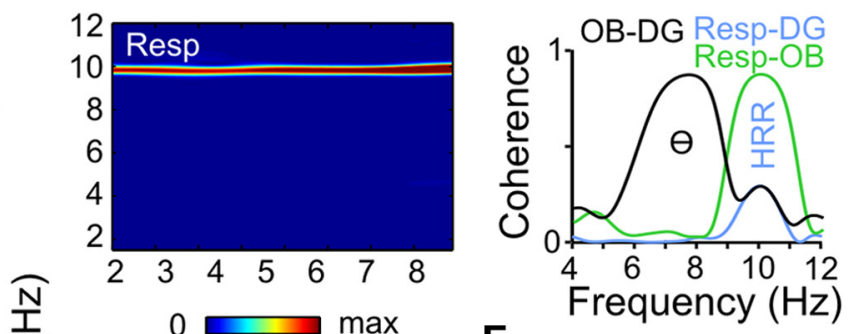

F

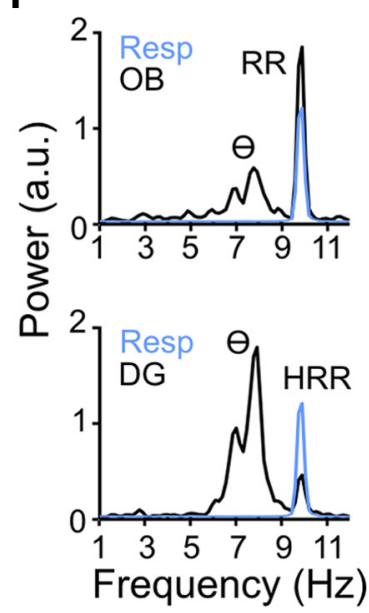

Figure 3. HRR can be slower or faster than theta $(\theta)$ during treadmill running depending on respiration rate. $\boldsymbol{A}-\boldsymbol{F}$, Same as in Figure 2 , but for animals recorded during treadmill running. $\boldsymbol{A}-\boldsymbol{C}$, Example in which HRR is slower than theta. $\boldsymbol{D}-\boldsymbol{F}$, Example in which HRR is faster than theta. In both examples, DG and OB LFPs have coherence peaks at RR and theta frequencies, whereas coherence between respiration and either DG or OB LFP is restricted to RR frequency. ex, Expiration; in, inspiration.

fied (RHA2116 or RHD2132, Intan Technologies), digitized (2.5 $\mathrm{kHz}$ ), and stored for offline analyses with custom written MATLAB routines (The MathWorks).

Juxtacellular recordings of hippocampal neurons in head-fixed awake mice were obtained with high-impedance quartz-glass micropipettes (o.d. 1.0/i.d. $0.5 \mathrm{~mm}$; impedance: $20-30 \mathrm{mOhm}$ ) pulled with the P-2000 puller (Sutter Instruments). Recording electrodes were filled with $1 \mathrm{M}$ sodium chloride and slowly lowered at 18 degree to the brain surface in 5 $\mu \mathrm{m}$ steps using the Micropositioner 2660 (Kopf Instruments). An Axoclamp 900A amplifier (Molecular Devices) in current-clamp mode with a 
A
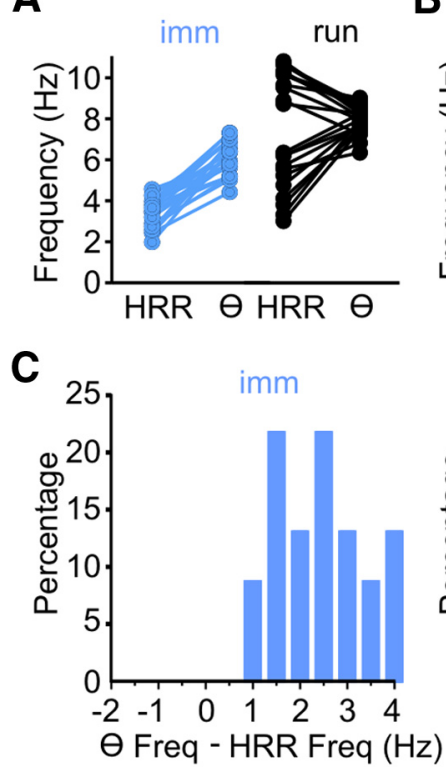

of the mean vector over all phases projected on the unit circle: $r=\| 1 / \mathrm{N}$ $\sum \exp \left(i \phi_{j}\right) \|$, where $\phi_{j}, j=1: \mathrm{N}$ are the phases associated with spikes and $i$ is the imaginary number. The statistical significance of spike-phase modulation was assessed through the Rayleigh test for uniformity of phase distribution; $p<0.05$ was considered statistically significant.

Depth profiles of theta and respiration-induced oscillations. We selected stable periods of theta and respiration-driven oscillations in which the peak frequencies of theta and respiration were separated by $>0.5$ Hz. For isolation of respiration-driven cycles, the signals were filtered (the precise cutoff depended on respiration rate and theta power in the power spectral density). Recordings from the hippocampal fissure served as reference for theta (Brankačk et al., 1993), whereas recordings from the hilus were used as reference for the HRR and gamma (Yanovsky et al., 2014). Peaks of identified cycles of theta at the hippocampal fissure or the respiratory oscillations at the hilus served as triggers for averaging the filtered signals across the 16 electrode positions of the silicon probes ( $n>30$ waves). Similarly, after filtering in the gamma frequency range $(40-100 \mathrm{~Hz})$, peaks of identified gamma cycles in the hilar reference channel were used as triggers for averaging gamma-filtered signals across the 16 electrode positions ( $n>50$ waves). Voltage profiles as a function of recording depth were first computed from the averaged evoked potentials in response to perforant pathway stimulation. These signals were subsequently used to estimate probe location (Brankačk et al., 1993; Yanovsky et al., 2014) (see Figs. 1B, 6C). Granule cell layers were localized by the polarity reversal of perforant path-evoked potentials. The hilus was estimated at the middle between both granule cell layers. In addition, the hippocampal fissure was identified from the amplitude maximum of theta, and the CA1 pyramidal cell layer from the $180^{\circ}$ theta phase reversal. Voltage profiles from averaged theta and respiration-driven oscillations were used to compute current source density as previously described (Brankačk et al., 1993).

Granger causality. We computed Granger causality spectra to assess frequency-specific directional influences between $\mathrm{OB}$ and hippocampal LFPs. In brief, Granger causality is based on autoregressive modeling and measures how much the past of a time-series $\mathrm{Y}$ predicts the present of another time series $\mathrm{X}$ beyond what is already accounted for by the past of $\mathrm{X}$ alone. Granger causality spectra was obtained through the MVGC MATLAB toolbox described by Barnett and Seth (2014), which is freely available online (http://users.sussex.ac.uk/ lionelb/MVGC/). Before assessing Granger causality, LFPs were down sampled by a factor of 10 (i.e., to a sampling rate of $250 \mathrm{~Hz}$ ). For all recordings, we used a fixed model order of 40, which was above the Akaike Information Criterion and provided a good frequency resolution with feasible computational cost.

Histology. After the conclusion of the experiments, the animals were deeply anesthetized with isoflurane and perfused transcardially with PBS and subsequently with 4\% PFA. Brains were carefully dissected, stored in PFA overnight, and coronal sections were cut $(50 \mu \mathrm{m})$, mounted, and stained with cresyl violet. The position of single electrodes and multichannel electrode probes was then verified by light microscopy.

Statistics. Data are expressed as mean \pm SEM. For group comparisons of normally distributed data (Kolmogorov-Smirnov test), we used $t$ test or repeated-measures ANOVA followed by Tukey's multiplecomparison test. For data with non-Gaussian distribution, we used the nonparametric Mann-Whitney test or the Wilcoxon signed rank test.

\section{Results}

The mouse hippocampus exhibits a respiration-entrained rhythm during awake immobility

We obtained LFPs from the OB and hippocampus of awake mice. The estimation of electrode location within the hippocampus was based on stereotaxic coordinates, histology (Fig. 1A), and characteristic anatomical landmarks in responses to perforant path stimulation (Fig. 1B) (Brankačk et al., 1993). Instantaneous respiration rate strongly depends on animal's behavior and is thus very variable in freely moving mice (Fig. 1D) (Kepecs et al., 2007). We therefore focused the present study on head-fixed animals because the behav- 
A

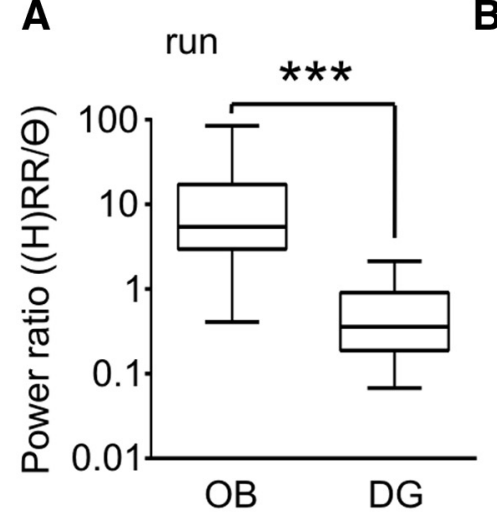

C

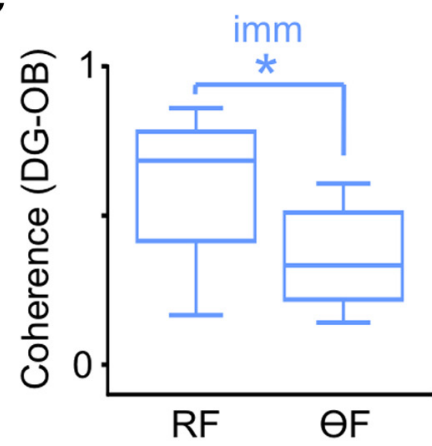

D

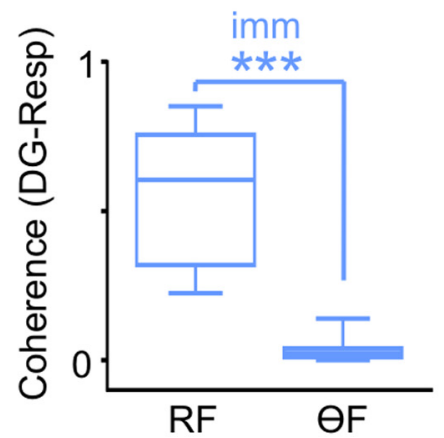

B
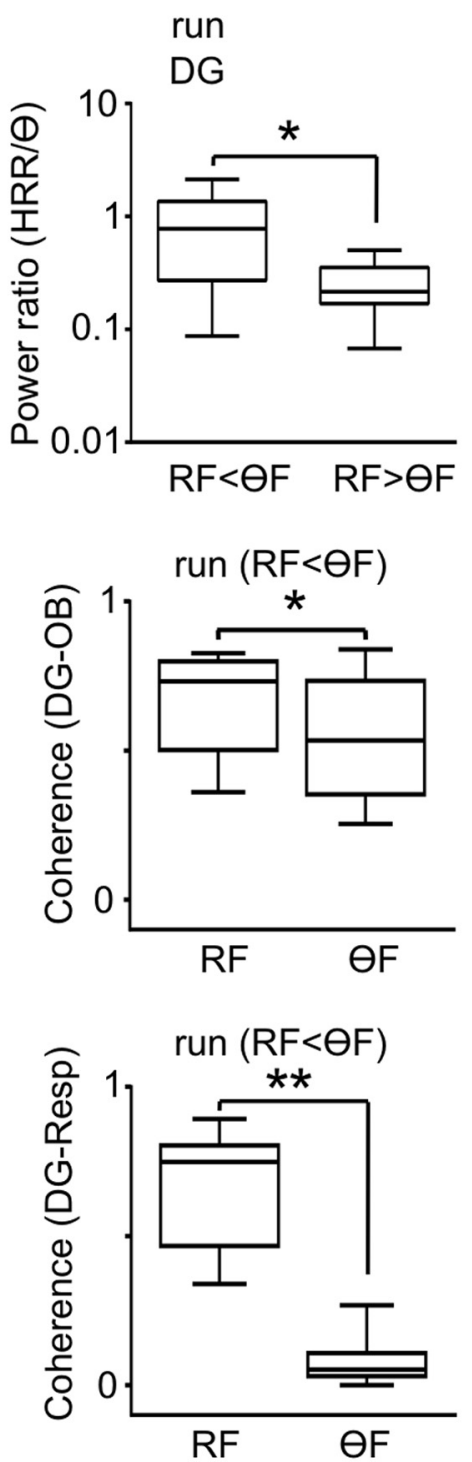
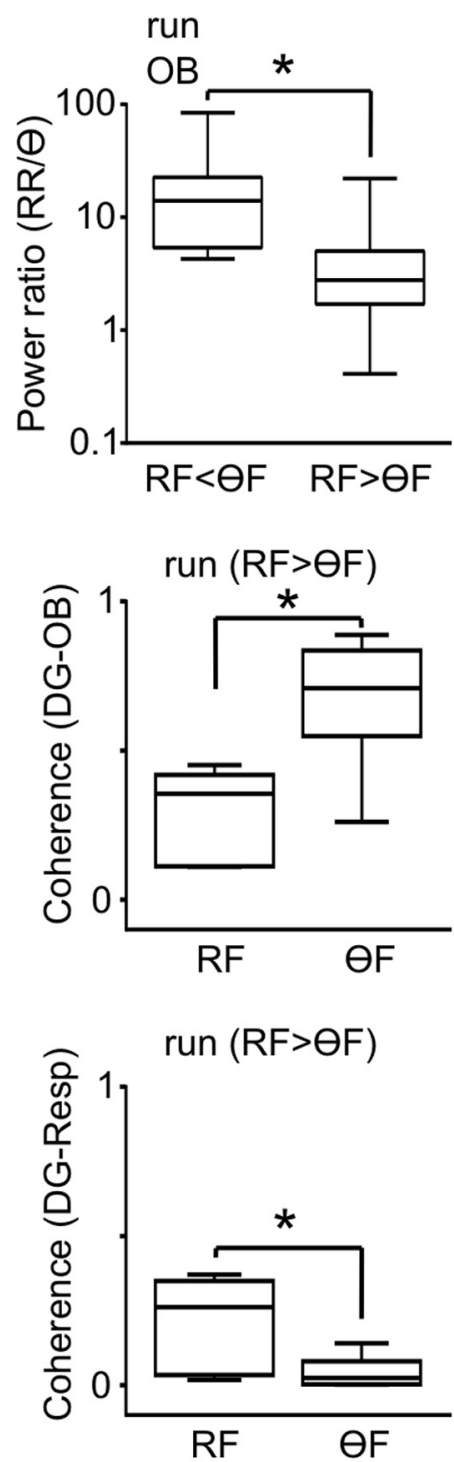

Figure 5. Power and coherence of HRR and theta oscillations depend on behavior. $A$, During running, the power ratio of RR to theta in OB is significantly larger than the ratio of $H R R$ to theta in $D G\left(n=18 ;{ }^{*} p<0.0001\right.$, Mann-Whitney test). $\boldsymbol{B}$, The power ratios of HRR (DG LFP, left) and RR (OB LFP, right) to theta are significantly larger when respiration frequency (RF) is lower than theta frequency $(\theta F)$ compared with when $\mathrm{RF}$ is higher than $\theta \mathrm{F}$ during running $\left(n=11\right.$ for $\mathrm{RF}<\theta \mathrm{F}, n=9$ for $\mathrm{RF}>\theta \mathrm{F} ;{ }^{*} p<0.05$, Mann-Whitney test; for examples, see Fig. $\left.3 C, F\right)$. C, $\boldsymbol{D}$, Coherence between DG and OB LFPs $(\boldsymbol{C})$ and between DG LFP and respiration $(\boldsymbol{D})$ is significantly larger at RF compared with $\theta$ F during both immobility $\left(n=18,{ }^{* * *} p<0.0001\right.$, Wilcoxon signed rank test $)$ and running when $\mathrm{RF}<\theta \mathrm{F}\left(n=10,{ }^{* *} p<0.001\right.$, Wilcoxon signed rank test). When $\mathrm{RF}>\theta \mathrm{F}$ during running, coherence between $\mathrm{DG}$ and $\mathrm{OB} \mathrm{LFPs}$ at $\theta \mathrm{F}$ is significantly larger than at $\mathrm{RF}\left(n=7,{ }^{*} p<\right.$ 0.05 , Wilcoxon signed rank test).

ior is basically clamped (to either immobility or running) and respiration is much more stable (Fig. $1 E$ ). Mice could be either forced (by means of a motor) or allowed to run freely over a circular treadmill positioned underneath (Fig. 1C). Periods with no treadmill rotation and no accelerometer signal were defined as immobility. We simultaneously recorded running speed and nasal respiration, the latter through a thermocouple located in front of the nostrils or chronically implanted into the nasal cavity.

The stable periods of respiration rate obtained under our experimental protocol allowed for fine-grained spectral analyses of respiratory and electrophysiological signals. During immobility, we found that awake animals express prominent LFP oscillations in the DG at the same frequency as respiration (Fig. $2 A$ ). The HRR was phase-locked to the RR seen in OB LFPs as well as with the respiration signal captured by the thermocouple (Fig. $2 B$ ). This finding distinguishes the present slow oscillation pattern in hippocampus
(HRR) from slow oscillations typical of up-and-down cortical states (Steriade et al., 1993), which are characteristically not coupled to respiration (Viczko et al., 2014; see also Lockmann and Belchior, 2014). Moreover, when looking at longer time scales of variable breathing activity, we found that changes in HRR peak frequency always matched changes in respiration rate (Figs. 2, 3).

Two types of hippocampal theta have been characterized based on their distinct pharmacology and behavioral correlates. Type I theta has somewhat higher frequency $(6-12 \mathrm{~Hz})$, occurs during running or locomotion (Vanderwolf, 1969), and is sensitive to urethane but resistant to atropine. Type II theta has lower frequency $(5-9 \mathrm{~Hz})$ and is sensitive to atropine but resistant to urethane (Kramis et al., 1975). In nonanesthetized animals, Type II theta is also known as "immobility theta" because it occurs during aroused immobility (Sainsbury and Montoya, 1984), which is a common state of headfixed animals. To differentiate HRR from Type II theta, we next 
A

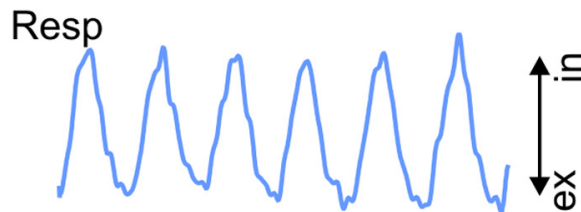

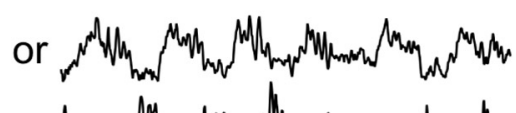
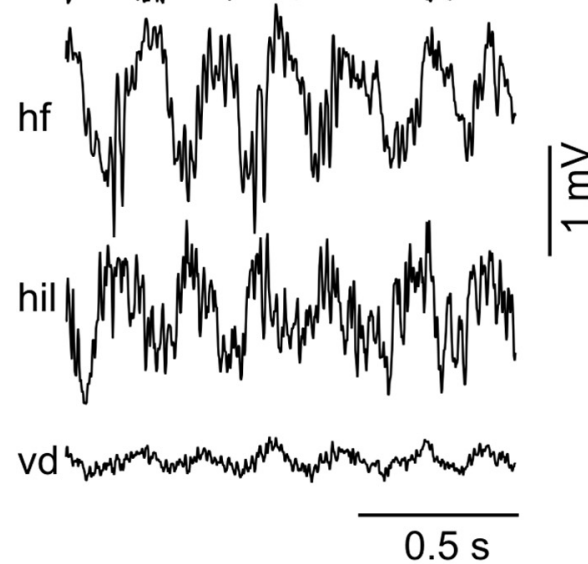

B

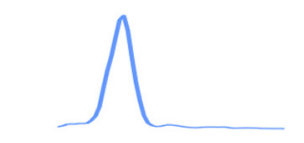

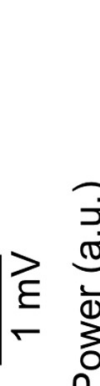

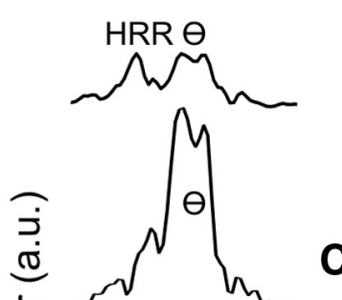

C
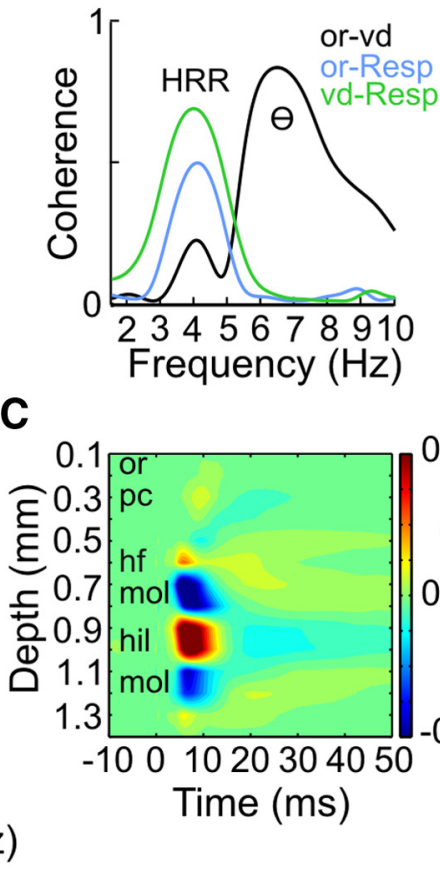
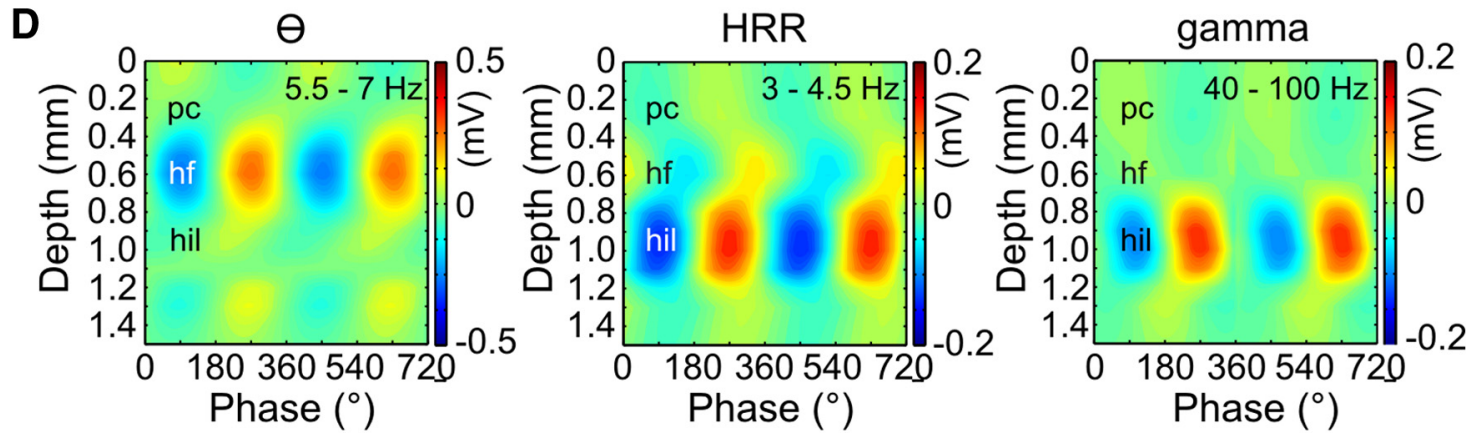

Figure 6. Laminar profile of LFP oscillations across the CA1-DG axis during immobility. A, Raw signals (left) and power (right) of respiration (Resp, blue), and of LFPs from stratum oriens (or), hippocampal fissure (hf), hilus (hil), and the ventral blade of DG (vd). B, Coherence spectra between or LFP and Resp (blue), between vd LFP and Resp (green), and between or and vd LFPs (black). C, Current source density analysis of evoked LFPs to stimulation of perforant pathway indicates anatomical landmarks used for probe localization. $\boldsymbol{D}$, Laminar voltage profiles of theta ( $\theta$, left), HRR (middle), and gamma (right). The amplitude maxima of theta and HRR occur in different layers, whereas HRR has a similar laminar profile as gamma oscillations. pc, Pyramidal cell layer; mol, molecular layer; ex, expiration; in, inspiration.

searched for periods of typical theta activity during head-fixed immobility. Figure $2 D-F$ shows an animal with a relatively high respiration frequency $(\sim 4.5 \mathrm{~Hz})$, reflecting increased arousal during immobility. As above, we found HRR in DG and RR in OB (as judged by the strong coherence with respiration; Fig. $2 E$ ). In addition to the HRR power peak at $\sim 4.5 \mathrm{~Hz}$, the DG LFP exhibited a second power peak $\sim 7 \mathrm{~Hz}$ (Fig. $2 F$ ). Time-frequency power decomposition (Fig. 2D) revealed that both rhythms coexisted simultaneously (this cannot be inferred by the power spectral density alone). Further analysis showed that the faster power peak corresponded to theta oscillations, as judged by the phase reversal across hippocampal layers and largest amplitude at the hippocampal fissure (data not shown for this example, but see Fig. 6) (Brankačk et al., 1993; Yanovsky et al., 2014). Despite the fact that the OB LFP exhibited low theta power, coherence between DG and OB peaked at both RR and theta frequencies (Fig. 2E). In stark contrast, there was no coherence between either OB or DG LFPs and the nasal thermocouple signal at theta frequency (for group results, see Fig. 5D).
HRR during treadmill running can be slower or faster than theta

Oscillation patterns and network synchrony depend on behavioral state. We next analyzed periods of treadmill running in head-fixed animals. Peak respiration rate was significantly $(n=$ $21 ; p<0.0001$, Wilcoxon signed rank test) higher during running (range: 3-10; median: $7.5 \mathrm{~Hz} ; n=21$ ) compared with immobility (range: $2-4.6 \mathrm{~Hz}$; median: $3.8 \mathrm{~Hz} ; n=23$ ). Similar to immobility, we could find clear periods of HRR during running, defined as a DG LFP activity with power peak at the same frequency as respiration and presenting high coherence with the nasal thermocouple signal (Fig. 3). As expected, theta oscillations were also prominent in DG during running; contrary to HRR, however, theta was not coherent with respiration (Fig. $3 B, E$ ). Interestingly, during running respiration and HRR could be slower (Fig. $3 A-C$ ) or faster (Fig. 3D-F) than theta.

Figure $4 A-C$ shows a statistical overview of the frequency variability of both respiration and theta oscillations. In our dataset, 
A
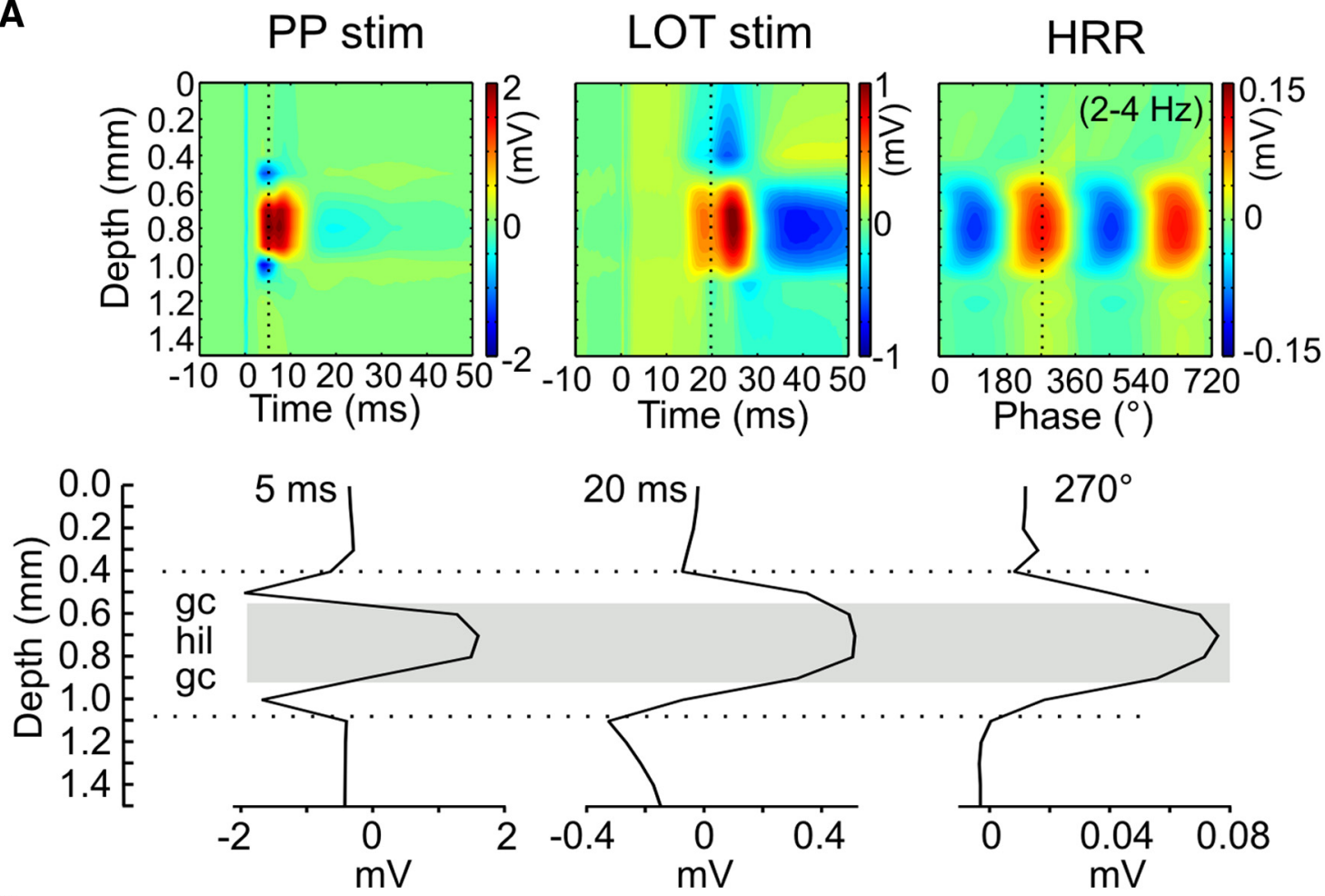

B
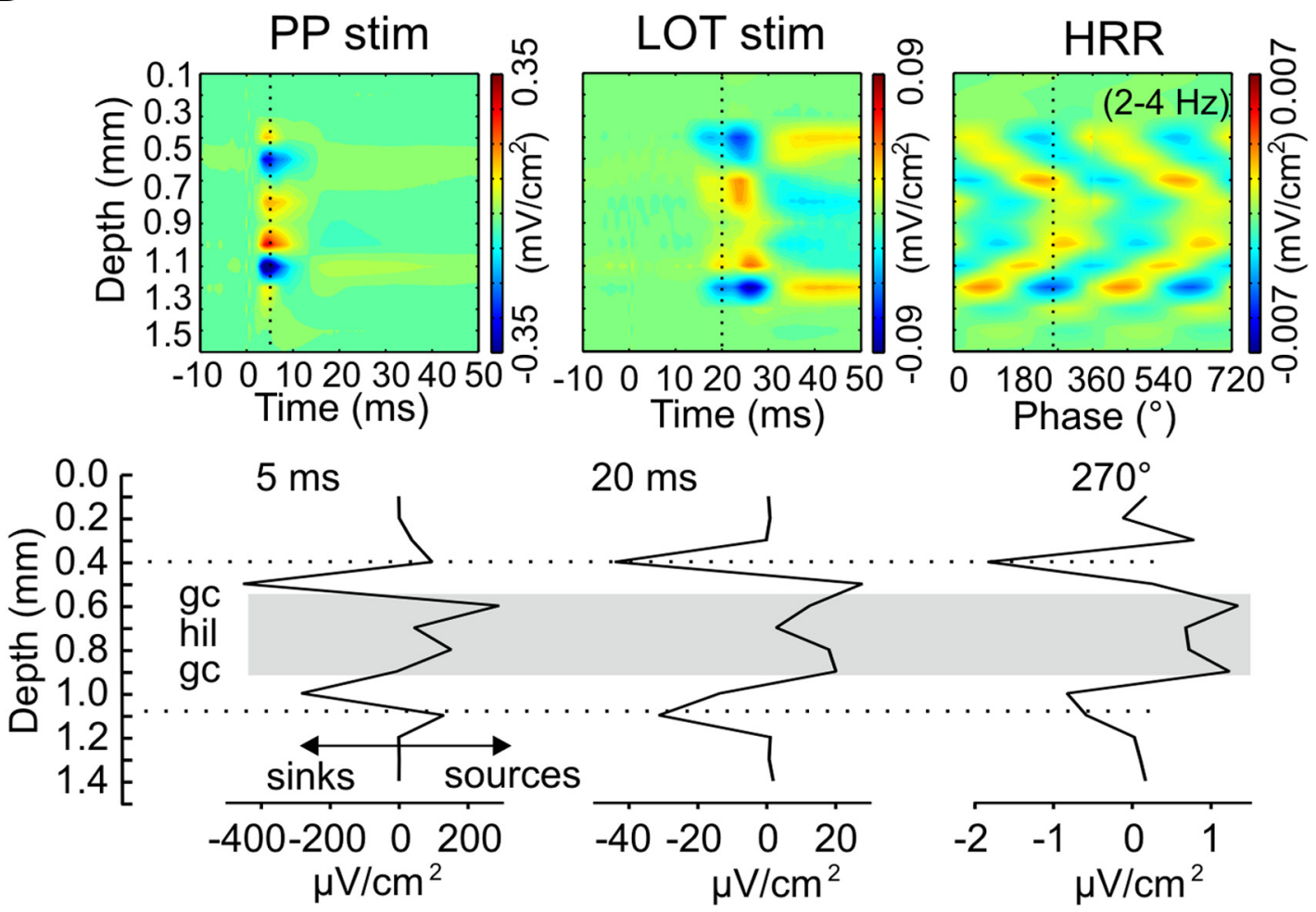

Figure 7. HRR has similar laminar profile to evoked responses after lateral olfactory tract (LOT) stimulation. $\boldsymbol{A}$, Laminar distribution of voltage at 5 ms after perforant pathway (PP) stimulation (left), at $20 \mathrm{~ms}$ after LOT stimulation (middle) and at the peak $\left(270^{\circ}\right.$ ) of HRR (right). Polarity reversal of the responses evoked by PP stimulation correspond to the granular cell layers ( $\mathrm{gc}$ ) and identify the extent of the hilus (hil, gray area). All three laminar profiles show maximum voltage in the hilus. $\boldsymbol{B}$, Laminar distribution of current source density. Note the precise correspondence between current sinks of HRR and induced by LOT stimulation in the outer molecular layer of the DG (dotted line).

the range of respiration frequencies was significantly larger than theta frequency range $(n=24 ; p<0.05$, Kolmogorov-Smirnov test). In addition, respiration frequency varied significantly less in immobility than during running $(n=23$ and $n=24 ; p<$
0.001 , Kolmogorov-Smirnov test), whereas variation of theta frequency was not different between immobility and running. We note that, for technical reasons, differential analysis of both rhythms required a minimal difference of $0.5 \mathrm{~Hz}$ in peak fre- 
A

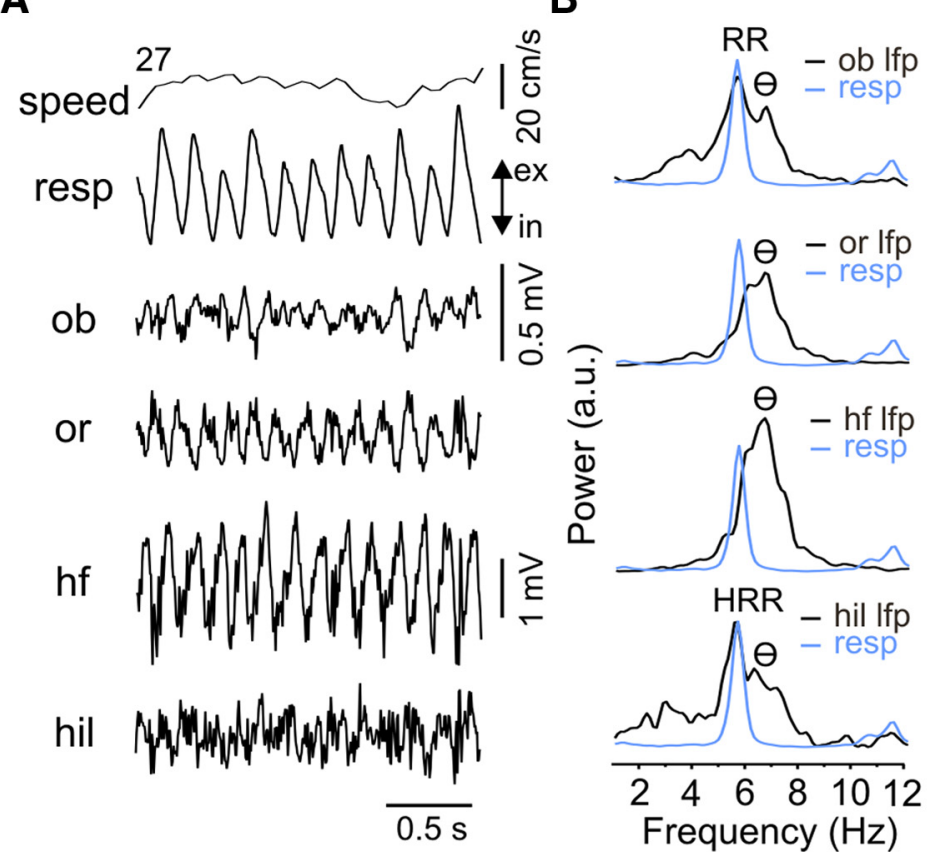

C
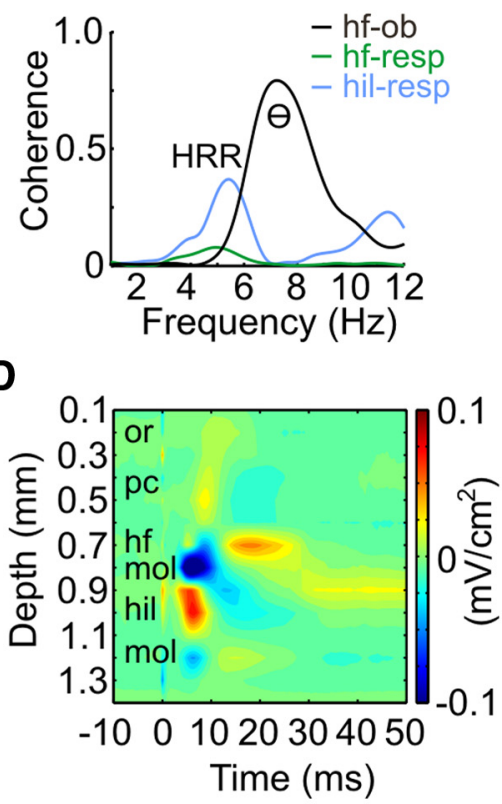

E

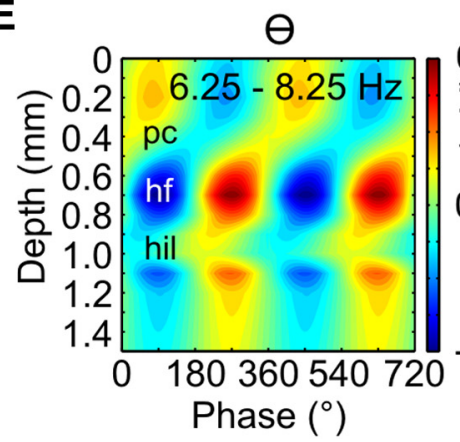

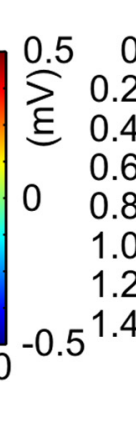

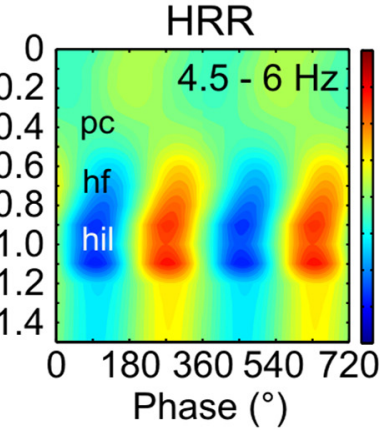

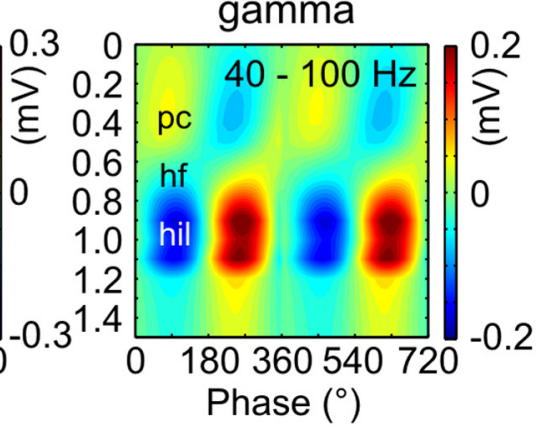

Figure 8. Laminar profile of LFP oscillations across the CA1-DG axis during running. $\boldsymbol{A}, \boldsymbol{B}$, Raw signals $(\boldsymbol{A})$ and power ( $\boldsymbol{B}$ ) of respiration (Resp, blue), and of LFPs from OB (ob), stratum oriens (or), hippocampal fissure (hf), and hilus (hil). Running speed is shown in $\boldsymbol{A}$ (top; initial speed: with $27 \mathrm{~cm} / \mathrm{s}$ ). C, Coherence spectra between hil LFP and Resp (blue), between hf LFP and Resp (green), and between hf and ob LFPs (black). D, Current source density analysis of evoked LFPs to stimulation of perforant pathway indicates anatomical landmarks used for probe localization. $E$, Laminar voltage profiles of theta ( $\theta$, left), HRR (middle), and gamma (right). Notice similar laminar profiles as found during immobility (Fig. 6), despite some mutual contamination of HRR-and theta-filtered LFPs due to the frequency overlap of HRR and theta in this example (see power spectra in $\boldsymbol{B}$ ). pc, Pyramidal cell layer; mol, molecular layer; ex, expiration; in, inspiration.

quency. Therefore, periods with overlapping frequencies were excluded from our analysis. This accounts for the gap between respiration rates higher and lower than theta frequency in our sample (Fig. $4 A, C$ ).

As expected, both respiration rate $(n=23$ and $n=24 ; p<$ 0.0001 , Mann-Whitney test) and theta frequency $(n=23$ and $n=$ $24 ; p<0.0001$, unpaired $t$ test) were significantly higher during running compared with immobility (Fig. 4A,B). Within immobility periods (Fig. $4 A, B$, blue), respiration rate was significantly lower than theta frequency $(n=23, p<0.0001$, paired $t$ test), whereas during running the average frequencies of respiration and theta were not statistically different (Fig. $4 A, B$, black).

\section{Two distinct slow oscillations are synchronously present in $\mathrm{OB}$ and hippocampus}

Surprisingly, during running the $\mathrm{OB}$ exhibited not only the respiration-coupled rhythm (RR) but also a second power peak at the same frequency as hippocampal theta oscillations (Fig. $3 A, D$, middle panels). Indeed, the power spectral density of OB and DG was similar, but with a reversed power relation: theta had lower power than RR in OB, but higher power than HRR in DG (Figs. $3 C, F, 5 A)$.

During running, the power ratio of HRR to theta in DG was significantly larger when respiration rate was below theta frequency compared with periods with respiration faster than theta (Fig. $5 B$, left; $n=11$ and $n=9 ; p<0.05$, Mann-Whitney test). In other words, HRR power peak was more prominent during running if respiration was slower than theta. A similar result was found for OB LFPs (Fig. $5 B$, right; $n=11$ and $n=9 ; p<0.05$, Mann-Whitney test).

Coherence between $\mathrm{OB}$ and DG peaked at the frequencies of respiration as well as theta (Fig. $3 B, E$, black line). This long-range coherence was significantly higher at the respiration frequency than at theta when respiration was slower than theta (Fig. $5 C$, left two panels; immobility: $n=18 ; p<0.0005$, Wilcoxon signed rank test; running: $n=10 ; p<0.05$, Wilcoxon signed rank test), but significantly higher at theta when respiration was faster (Fig. $5 C$, right; $n=$ $7 ; p<0.05$, Wilcoxon signed rank test). Of note, the absolute difference between the peak frequencies of theta and HRR was similar in running periods with respiration slower and faster than theta $(p=$ 0.68 , Mann-Whitney test), which therefore does not account for the 


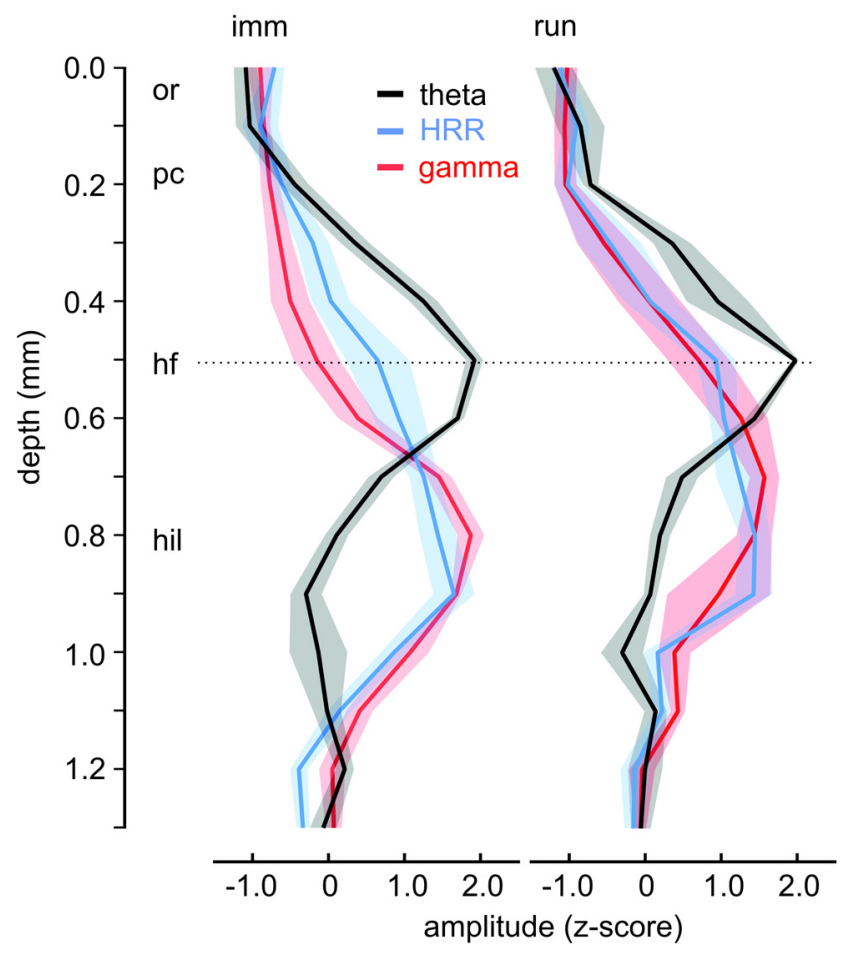

Figure 9. Laminar profiles of HRR, theta and gamma (group results). Depth-voltage values were $z$-scored to account for $1 / f$ and allow comparing different frequencies. Thick lines indicate the mean. Shaded areas represent SEM. For each frequency, the group averages are not significantly different (multiple $t$ tests using the Holm-Sidak method, $p>0.05$ ) between immobility (imm, $n=6$ ) and running (run, $n=5$ ). Whereas theta has the maximum at the hippocampal fissure ( $h f$ ), both gamma and HRR are maximal in the hilus (hil). or, Stratum oriens; pc, pyramidal cell layer.

observed changes in coherence levels. Coherence between LFPs and the respiration signal was always high at respiration frequency and low in the theta domain, regardless of the frequency difference between theta and respiration (Fig. 5D).

In all, these results show that two distinct slow rhythms with overlapping frequencies in the range between 2 and $12 \mathrm{~Hz}$ are simultaneously present in the hippocampus and $\mathrm{OB}$ of awake mice. The power relationship of the two oscillations depends on behavior and brain structure. Our results also show that respiration-coupled and theta oscillations can occur coherently between the $\mathrm{OB}$ and hippocampus.

\section{Different laminar profiles of HRR and theta}

Laminar profiles of field potential signals can be used to identify distinct mechanisms underlying specific activity patterns. To delineate both rhythms, we recorded amplitude-depth profiles of HRR and theta in awake, head-fixed mice. Using linear probes of 16 channels across the CA1-DG axis (interelectrode distance: $100 \mu \mathrm{m}$ ), we found that the depth profiles for theta and HRR are clearly different (see Yanovsky et al., 2014): the phase of theta reversed below the pyramidal cell layer, and theta amplitude peaked at the fissure; on the other hand, HRR exhibited a much more modest phase shift and was maximal at the hilus. Figure 6 shows a representative example of aroused immobility with theta. HRR has a similar depth profile as gamma oscillations (Fig. 6D, right), which are well known to be highest at the hilus (Bragin et al., 1995; Caixeta et al., 2013).

The amplitude of theta and HRR is low in LFPs recorded far above the fissure or below the hilus. Interestingly, however, in LFP recordings at far sites targeting superficial and deep hippocampal layers, both rhythms appear in approximately equal amplitude relative to each other. Figure $6 A$ illustrates this finding with representa- tive LFPs from stratum oriens and from the ventral DG blade (see also Yanovsky et al., 2014). HRR recorded from either site was coherent with the respiration signal, whereas theta was not (Fig. 6B, green and blue lines). As expected, superficial and deep hippocampal electrodes were coherent not only at HRR but also at theta frequency (Fig. 6B, black line).

In two mice with a probe location centered at the DG, we recorded evoked responses to stimulation of the perforant pathway and LOT and could observe that the amplitude of the voltage responses varied with depth and was maximal in the hilus in either case, thus resembling HRR laminar profile (for the results of one such animal, see Fig. 7). Interestingly, current source density analysis revealed that the current sinks of HRR exactly matched the current sinks of responses to LOT stimulation in the outer molecular layers of DG (Fig. 7, dotted lines), whereas the current sinks after perforant path stimulation were located closer to the granular cell layers.

Depth profiles for HRR and theta during running were identical to immobility (Fig. 8; for group results, see Fig. 9). In all, the results show that theta and HRR have different laminar profiles across the hippocampus of awake mice.

\section{HRR modulates neuronal spiking}

We next searched for coupling between spike times and the phase of HRR. Juxtacellular recordings revealed that $75 \%$ of units in both CA1 and DG are modulated by HRR alone, by theta alone, or by both theta and HRR (Fig. 10C). In DG, from a total of 32 juxtacellularly recorded units, 4 cells $(12.5 \%)$ were modulated only by HRR, $12(37.5 \%)$ only by theta, and $8(25 \%)$ by both oscillations (Fig. 10D). Figure $10 \mathrm{~A}$ shows examples of the neuronal spiking of these three cell types, along with the respiration signal and the DG LFP. Spiking probability over the phases of HRR and theta is shown in Figure $10 B$.

Interestingly, the specificity of spike modulation differed in CA1, where no unit was modulated by HRR alone: 6 of 23 cells (26\%) were modulated only by theta and $11(48 \%)$ were modulated by both oscillations (Fig. 10D). We did not find differences in the strength of spike-phase coupling between juxtacellularly recorded CA1 and DG units, either by theta or by HRR (Fig. 10E).

Together, the results show that the respiratory cycle modulates spiking probability not only in classical olfactory regions, such as the OB (Buonviso et al., 2003), but also in more multimodal brain regions, such as the hippocampus (see also Ito et al., 2014; Tsanov et al., 2014).

\section{OB and DG mutually drive each other at different frequency channels}

Finally, we performed directionality analyses between simultaneously recorded LFPs in OB and DG in awake animals during both behavioral states: immobility and running. Directional influences were assessed by Granger causality in the frequency domain (Barnett and Seth, 2014; Seth et al., 2015), which measures, at each frequency, the variance of a recorded region that is explained by past recordings of another region beyond what is explained by its own past (Ding et al., 2006).

Granger causality spectra revealed that OB LFPs had causal influences on the HRR recorded in DG (Fig. 11). Interestingly, the hippocampus also exerted Granger causal influence on the $\mathrm{OB}$, but this influence was prominent at theta frequency (Fig. $11 A, B$, right panels). In contrast, Granger causality from $O B$ to $D G$ at theta frequency and from $D G$ to $O B$ at respiration frequency were much weaker (Fig. $11 A, C)$. Thus, the results suggest that HRR is driven by the $\mathrm{OB}$ and that the $\mathrm{OB}$ theta is driven by the hippocampus (Fig. 11D). 
A
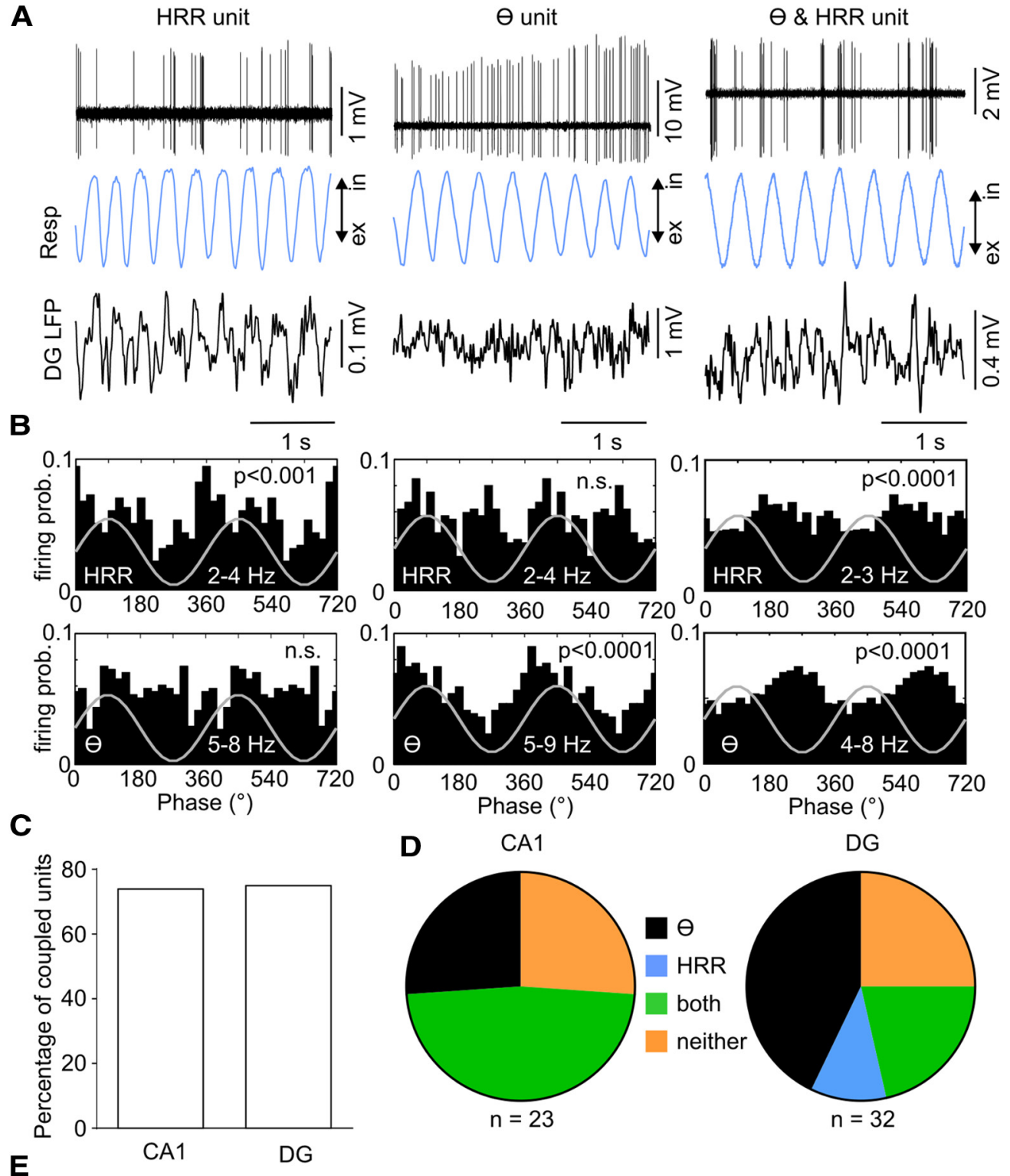

D

CA1
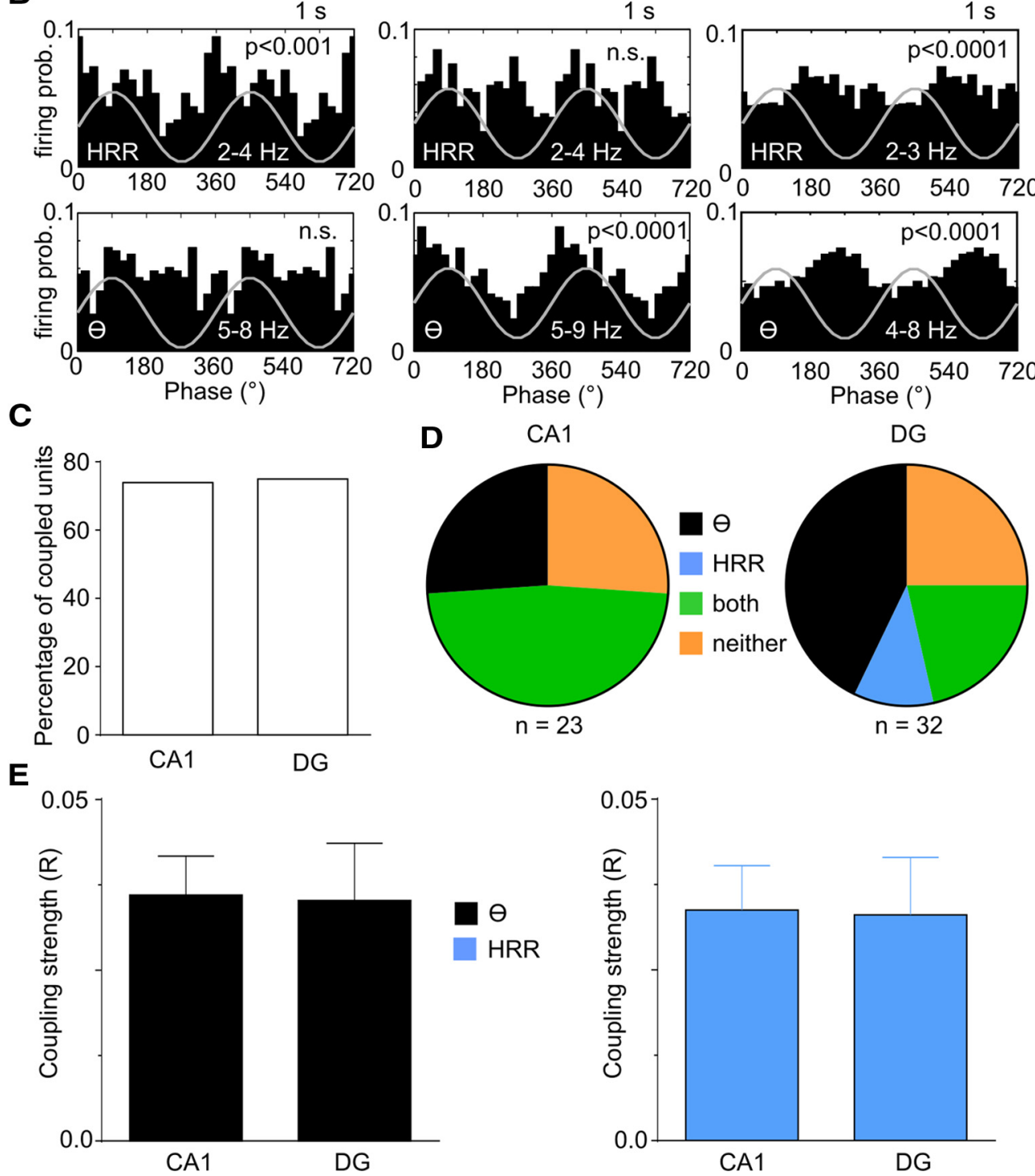

Figure 10. HRR modulates spiking activity in awake animals. $A$, Examples of juxtacellularly recorded discharges (top), respiration (Resp, blue) and DG LFP (black) for units coupled only to HRR (left), only to theta ( $\theta$, middle), or to both rhythms (right). $\boldsymbol{B}$, Spike distribution over the phases of HRR (top) and of theta (bottom) for the same cells as in $\boldsymbol{A}$. n.s., Not significant. $\boldsymbol{C}, \boldsymbol{D}$, Percentage of cells significantly coupled to either theta or HRR in CA1 and DG $(\boldsymbol{C})$, and distribution of coupling selectivity (D). $\boldsymbol{E}$, Average coupling strength to theta (black) and HRR (blue) for units recorded in CA1 and DG.

\section{Discussion}

In the present study, we demonstrate the existence of a slow oscillation in the hippocampus of awake mice, which is highly coherent with nasal respiration. Importantly, the HRR is distinct from the pattern of theta oscillations: First, the power of HRR is maximal at the hilus, whereas theta oscillations peak at the hippocampal fissure. Second, the typical $180^{\circ}$ phase shift of theta across the CA1 pyramidal cell layer is absent for HRR. Third, only 
A
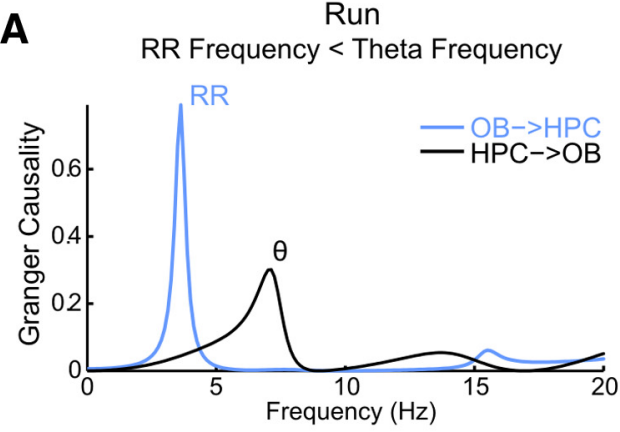

\section{B}

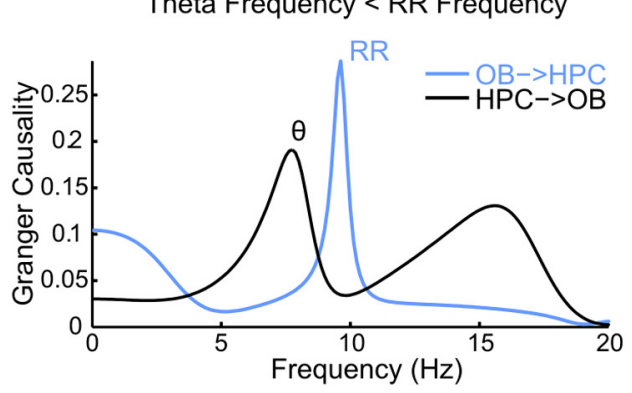

C

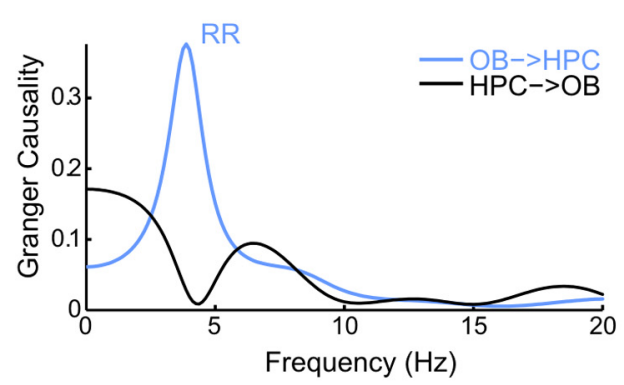

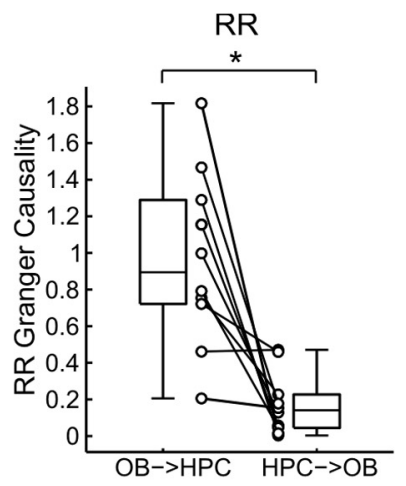
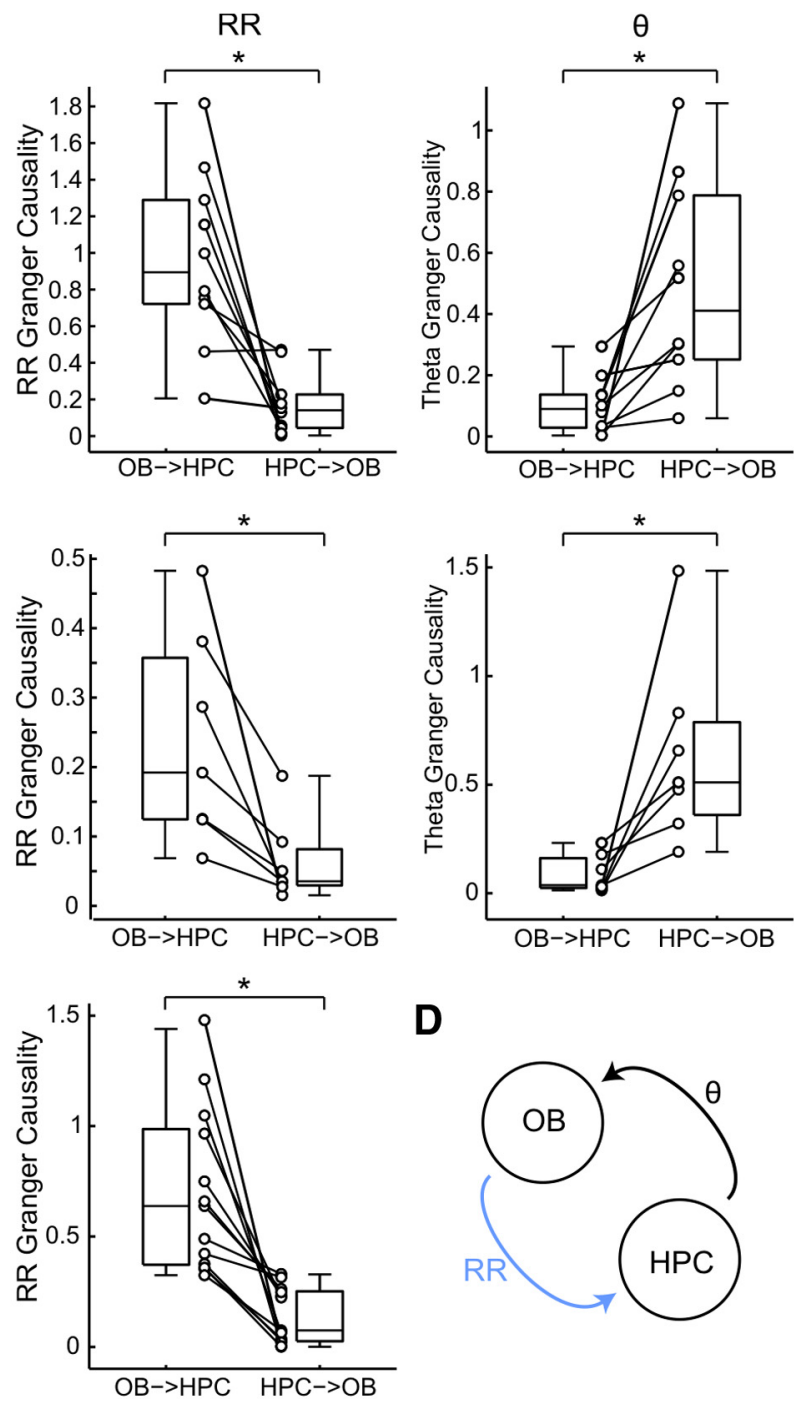

D

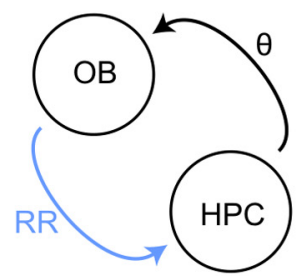

Figure 11. Directionality analysis reveals that the $O B$ causes the RR in the hippocampus, whereas the hippocampus causes theta $(\theta)$ in $0 B$. $\boldsymbol{A}, \boldsymbol{B}$, Left, Examples of Granger causality spectra for $L F$ s recorded in $0 B$ and hippocampus (HPC) during treadmill running in which RR was slower $(\boldsymbol{A})$ or faster $(\boldsymbol{B})$ than theta $\left(\boldsymbol{A}: n=10,{ }^{*} p<0.005\right.$ for either comparison; $\boldsymbol{B}$ : $n=7,{ }^{*} p<0.05$ for either comparison; Wilcoxon signed rank test). Notice in both cases a peak in $\mathrm{OB} \rightarrow \mathrm{HPC}$ causality at RR frequency and a peak in HPC $\rightarrow O B$ causality at theta frequency. Boxplots on right represent group results. $C$, Same as above but for LFP recordings during head-fixed immobility $\left(n=13,{ }^{*} p<0.0005\right.$; Wilcoxon signed rank test; theta causality was not analyzed because not all animals exhibited theta during immobility). D, Schematic depiction of RR and theta causal influences between $O B$ and $H P C$.

HRR but not theta is entrained by the respiratory cycle. In consequence, HRR peak frequency is much more variable than theta peak frequency. We further showed that discharges of neurons within DG and CA1 are modulated by either theta, HRR, or by both rhythms. These results significantly extend our recent findings in urethane-anesthetized mice (Yanovsky et al., 2014) to awake mice. Thus, respiration provides a rhythmic signal, which entrains neurons during long-range synchronization of olfactory and hippocampal networks. This mechanism likely contributes to context-dependent multimodal information processing in limbic networks (Butterly et al., 2012).

Respiration-entrained rhythm and theta oscillations share similar frequency bands. For reliable identification of both patterns, we recorded nasal respiration synchronously with LFPs, and we focused analysis on periods with different peak frequencies. Respiration rate in awake mice is highly variable, especially in freely moving mice during exploration when bouts of sniffing occur. We reduced breathing variability by working with a head-fixed preparation with basically two distinct patterns of behavior: awake immobility or treadmill running. The approximately steady respiration rate during either state allowed for spectral analysis with sufficient frequency resolution to sort apart theta and HRR.

During running, respiration frequency could overlap, be slower, or be faster than theta. In all recordings, however, HRR peak frequency matched the peak frequency of the respiration signal, and both these signals were coherent. Interestingly, HRR in DG was most prominent when respiration rate was lower than theta frequency, indicating that entrainment of downstream networks by respiration is rate-dependent. The underlying mechanisms are presently unclear but involve propagation of signals via the lateral olfactory tract (Yanovsky et al., 2014). As a corollary, our results imply that, without assessing respiration, it is difficult to untangle theta and HRR in LFP recordings, especially in the DG. This may justify revisiting multiple findings previously attributed to theta oscillations, which may have been "contaminated" by HRR.

Seminal reports provided evidence both for (Macrides, 1975; Macrides et al., 1982) and against (Vanderwolf and Szechtman, 
1987) the existence of an LFP rhythm entrained by nasal respiration in the rodent hippocampus during active sniffing. In reality, rather than searching for a separated rhythm, these investigators were studying whether the hippocampal theta rhythm (called RSA by the time, "rhythmic slow activity") could be entrained with sniffing. In this sense, Vanderwolf and Szechtman (1987) concluded that: "if hippocampal RSA and sniffing occur concurrently, the present results show that the two rhythms may differ in frequency by as much as $2 \mathrm{~Hz}$." However, Macrides (1975) and Macrides et al. (1982) found clear examples of theta and rhythmic sniffing at the same frequency and showed that both rhythms could be entrained. Unfortunately, the available tools for electrophysiological recordings and especially of digital signal analysis were rather poor at the time. We think that these important technical limitations have hindered recognizing and sorting apart two distinct hippocampal rhythms of very close frequency. Rather than entrainment of theta with sniffing, we think that Macrides may have actually detected HRR activity.

More recently, Kay (2005) reported in rats that the phase coherence between hippocampal and OB LFPs increases in the 6-12 $\mathrm{Hz}$ range during odor sampling in an olfactory discrimination task. However, because respiratory activity was not directly measured, whether the increase in theta-band coherence observed by Kay (2005) was due to a respiration-coupled rhythm or not is unclear. Macrides et al. (1982) and Kay (2005) interpreted the results as an entrainment of hippocampal Type 1 theta to sniffing, which would be relevant for sensorimotor integration. While our work does not discard the possibility that hippocampal theta may come to be entrained by sniffing, to the best of our knowledge, our results constitute the first demonstration that hippocampal Type 1 theta may coexist with a distinct LFP rhythm, which is coupled to nasal respiration. Furthermore, along with our recent findings in urethane-anesthetized mice (Yanovsky et al., 2014), there is now solid evidence to promote HRR as a clearly distinct pattern from hippocampal Type 2 theta as well.

Directionality analysis of simultaneously recorded LFPs in DG and OB revealed that HRR in DG could be predicted by the past of OB RR, which is to say that the OB Granger-caused HRR in DG. This result is consistent with our previous report showing that tracheotomy abolishes respiration-entrained rhythms in both OB (RR) and hippocampus (HRR) in anesthetized animals (Yanovsky et al., 2014; see also Fontanini et al., 2003). Hence, HRR is not directly generated by central pattern generators in the brainstem, such as the Pre-Bötzinger complex (Smith et al., 1991). Consistent with our causality analysis, there is much anatomical and physiological evidence for olfactory inputs reaching the DG via the lateral entorhinal cortex (Wilson and Steward, 1978; Wouterlood and Nederlof, 1983; Kerr et al., 2007), which is considered part of the olfactory cortex (Haberly and Price, 1978; Agster and Burwell, 2009; Sosulski et al., 2011). Olfactory afferents originating from the piriform cortex or from mitral cells in OB target layer I of the lateral entorhinal cortex, where they synapse on the apical dendrites of layer II/III cells (Luskin and Price, 1983; Burwell and Amaral, 1998). These in turn project to the hippocampal formation as well as back to olfactory regions (Agster and Burwell, 2009). In accordance to this scenario, HRR activity is associated with current sinks in the outer molecular layer of DG, where afferences from the lateral entorhinal cortex terminate (Amaral and Witter, 1989; Witter, 1993; Canning et al., 2000).

Interestingly, although RR dominated the oscillatory content of OB LFPs, the OB also exhibited some theta activity (reflected in the smaller peak in the power spectrum). Theta oscillations re- corded in OB were highly coherent with hippocampal theta oscillations, and, moreover, were Granger-caused by them. There are diverse connections between both regions, which may mediate this interaction. These include the common cholinergic input from medial septum and diagonal band of Broca, which reaches the entorhinal cortex and the hippocampal formation as well as OB and piriform cortex (Senut et al., 1989; Kerr et al., 2007). In addition, there are top-down connections from the lateral entorhinal cortex to the OB via the piriform cortex (Haberly and Price, 1978; Agster and Burwell, 2009; Xu and Wilson, 2012), which are likely to contribute to our Granger causality results.

Previous studies have referred to the respiration-entrained rhythm in OB LFPs as "theta" (Margrie and Schaefer, 2003; Kay, 2005; Rojas-Libano et al., 2014), probably motivated by the overlap between the frequency range of respiration and of hippocampal theta oscillations. Here we opted not to use the same definition because our results show that the $\mathrm{OB}$ can produce both a respiration-entrained rhythm and an LFP oscillation coupled to hippocampal theta, and we actually suggest restricting the definition of "OB theta" to the latter activity. In this sense, field potential oscillations in the broad theta frequency domain should be carefully dissected into respiration- and theta-driven components or termed neutrally by their frequency. The term "OB theta" may give a false impression of a unique, and somewhat global, theta rhythm, whereas, in contrast, here we demonstrate the existence of two clearly distinct LFP rhythms within the usual theta range.

The present results add to a myriad of findings suggesting that oscillations are important for the transfer of information within the brain (Buzsáki and Draguhn, 2004; Buzsáki, 2010, Fell and Axmacher, 2011), in this case, between sensory and memory networks. Rodents are "olfactory animals," and their brains seem to have evolved to oscillate at the same frequency as they sample environmental cues through their nostrils. Previous studies have already suggested that the respiratory cycle constitutes an important scaffold for information processing (Chaput, 1986; Buonviso et al., 2003; Fontanini and Bower, 2006; Kepecs et al., 2006). Interestingly, recent work on awake head-fixed mice has shown that respiration-entrained rhythms reach the barrel cortex (Ito et al., 2014). Our study now shows that respiration does even impinge on a further downstream region in awake mice, namely, the hippocampus. Together, these findings suggest that respirationentrained oscillations constitute a ubiquitous channel for network communications across the brain.

\section{References}

Adrian ED (1942) Olfactory reactions in the brain of the hedgehog. J Physiol 100:459-473. CrossRef Medline

Agster KL, Burwell RD (2009) Cortical efferents of the perirhinal, postrhinal, and entorhinal cortices of the rat. Hippocampus 19:1159-1186. CrossRef Medline

Amaral DG, Witter MP (1989) The three-dimensional organization of the hippocampal formation: a review of anatomical data. Neuroscience 31: 571-591. CrossRef Medline

Barnett L, Seth AK (2014) The MVGC multivariate Granger causality toolbox: a new approach to Granger-causal inference. J Neurosci Methods 223:50-68. CrossRef Medline

Battaglia FP, Benchenane K, Sirota A, Pennartz CM, Wiener SI (2011) The hippocampus: hub of brain network communication for memory. Trends Cogn Sci 15:310-318. CrossRef Medline

Bragin A, Jandó G, Nádasdy Z, Hetke J, Wise K, Buzsáki G (1995) Gamma $(40-100 \mathrm{~Hz})$ oscillation in the hippocampus of the behaving rat. J Neurosci 15:47-60. Medline

Brankačk J, Buzsáki G (1986) Hippocampal responses evoked by tooth pulp and acoustic stimulation: depth profiles and effect of behavior. Brain Res 378:303-314. CrossRef Medline 
Brankačk J, Stewart M, Fox SE (1993) Current source density analysis of the hippocampal theta rhythm: associated sustained potentials and candidate synaptic generators. Brain Res 615:310-327. CrossRef Medline

Buonviso N, Amat C, Litaudon P, Roux S, Royet JP, Farget V, Sicard G (2003) Rhythm sequence through the olfactory bulb layers during the time window of a respiratory cycle. Eur J Neurosci 17:1811-1819. CrossRef Medline

Burwell RD, Amaral DG (1998) Cortical afferents of the perirhinal, postrhinal, and entorhinal cortices of the rat. J Comp Neurol 398:179-205. CrossRef Medline

Butterly DA, Petroccione MA, Smith DM (2012) Hippocampal context processing is critical for interference free recall of odor memories in rats. Hippocampus 22:906-913. CrossRef Medline

Buzsáki G (2002) Theta oscillations in the hippocampus. Neuron 33: 325-340. CrossRef Medline

Buzsáki G (2010) Neural syntax: cell assemblies, synapsembles, and readers. Neuron 68:362-385. CrossRef Medline

Buzsáki G, Draguhn A (2004) Neuronal oscillations in cortical networks. Science 304:1926-1929. CrossRef Medline

Buzsáki G, Moser EI (2013) Memory, navigation and theta rhythm in the hippocampal-entorhinal system. Nat Neurosci 16:130-138. CrossRef Medline

Caixeta FV, Cornélio AM, Scheffer-Teixeira R, Ribeiro S, Tort AB (2013) Ketamine alters oscillatory coupling in the hippocampus. Sci Rep 3:2348. CrossRef Medline

Canning KJ, Wu K, Peloquin P, Kloosterman F, Leung LS (2000) Physiology of the entorhinal and perirhinal projections to the hippocampus studied by current source density analysis. Ann N Y Acad Sci 911:55-72. CrossRef Medline

Chaput MA (1986) Respiratory-phase-related coding of olfactory information in the olfactory bulb. Physiol Behav 36:319-324. CrossRef Medline

Colgin LL, Denninger T, Fyhn M, Hafting T, Bonnevie T, Jensen O, Moser MB, Moser EI (2009) Frequency of gamma oscillations routes flow of information in the hippocampus. Nature 462:353-357. CrossRef Medline

Delorme A, Makeig S (2004) EEGLAB: an open source toolbox for analysis of single-trial EEG dynamics including independent component analysis. J Neurosci Methods 134:9-21. CrossRef Medline

European Science Foundation (2001) Use of animals in research: European Science Foundation Policy Briefings, Ed 2. Strasbourg, France: European Science Foundation.

Ding M, Chen Y, Bressler SL (2006) Granger causality: basic theory and application to neuroscience. In: Handbook of time series analysis (Schelter B, Winterhalder M, Timmer J, eds), pp 451-474. New York: Wiley-VCH.

Fell J, Axmacher N (2011) The role of phase synchronization in memory processes. Nat Rev Neurosci 12:105-118. CrossRef Medline

Fontanini A, Bower JM (2006) Slow-waves in the olfactory system: an olfactory perspective on cortical rhythms. Trends Neurosci 29:429-437. CrossRef Medline

Fontanini A, Spano P, Bower JM (2003) Ketamine-xylazine-induced slow $(<1.5 \mathrm{~Hz})$ oscillations in the rat piriform (olfactory) cortex are functionally correlated with respiration. J Neurosci 23:7993-8001. Medline

Haberly LB, Price JL (1978) Association and commissural fiber systems of the olfactory cortex of the rat: I. Systems originating in the piriform cortex and adjacent areas. J Comp Neurol 178:711-740. CrossRef Medline

Igarashi J, Isomura Y, Arai K, Harukuni R, Fukai T (2013) A $\theta-\gamma$ oscillation code for neuronal coordination during motor behavior. J Neurosci 33: 18515-18530. CrossRef Medline

Ito J, Roy S, Liu Y, Cao Y, Fletcher M, Lu L, Boughter JD, Grün S, Heck DH (2014) Whisker barrel cortex delta oscillations and gamma power in the awake mouse are linked to respiration. Nat Commun 5:3572. CrossRef Medline

Kay LM (2005) Theta oscillations and sensorimotor performance. Proc Natl Acad Sci U S A 102:3863-3868. CrossRef Medline

Kay LM, Beshel J, Brea J, Martin C, Rojas-Líbano D, Kopell N (2009) Olfactory oscillations: the what, how and what for. Trends Neurosci 32: 207-214. CrossRef Medline

Kepecs A, Uchida N, Mainen ZF (2006) The sniff as a unit of olfactory processing. Chem Senses 31:167-179. Medline

Kepecs A, Uchida N, Mainen ZF (2007) Rapid and precise control of sniffing during olfactory discrimination in rats. J Neurophysiol 98:205-213. CrossRef Medline

Kerr KM, Agster KL, Furtak SC, Burwell RD (2007) Functional neuroanat- omy of the parahippocampal region: the lateral and medial entorhinal areas. Hippocampus 17:697-708. CrossRef Medline

Klingberg F, Pickenhain L, Gelbrich D (1964) Registrierung der Atmung bei der frei beweglichen Ratte im chronischen electrophysiologischen Experiment. Acta Biol Med Ger 12:619-622. Medline

Kramis R, Vanderwolf CH, Bland BH (1975) Two types of hippocampal rhythmical slow activity in both the rabbit and the rat: relations to behavior and effects of atropine, diethyl ether, urethane, and pentobarbital. Exp Neurol 49:58-85. CrossRef Medline

Lockmann AL, Belchior H (2014) New insights into the role of respiratory inputs in hippocampal oscillations. J Neurosci 34:9473-9475. CrossRef Medline

Luskin MB, Price JL (1983) The laminar distribution of intracortical fibers originating in the olfactory cortex of the rat. J Comp Neurol 216:292-302. CrossRef Medline

Macrides F (1975) Temporal relationships between hippocampal slow waves and exploratory sniffing in hamsters. Behav Biol 14:295-308. CrossRef Medline

Macrides F, Eichenbaum HB, Forbes WB (1982) Temporal relationship between sniffing and the limbic $\theta$ rhythm during odor discrimination reversal learning. J Neurosci 2:1706-1717. Medline

Margrie TW, Schaefer AT (2003) Theta oscillation coupled spike latencies yield computational vigour in a mammalian sensory system. J Physiol 546:363-374. CrossRef Medline

Miltner WH, Braun C, Arnold M, Witte H, Taub E (1999) Coherence of gamma-band EEG activity as a basis for associative learning. Nature 397: 434-436. CrossRef Medline

Montgomery SM, Buzsáki G (2007) Gamma oscillations dynamically couple hippocampal CA3 and CA1 regions during memory task performance. Proc Natl Acad Sci U S A 104:14495-14500. CrossRef Medline

National Research Council (1996) Guide for the care and use of laboratory animals. Institute of Laboratory Animal Research, Commission on Life Sciences. National Research Council. Washington, DC: National Academies.

Paxinos G, Franklin KBJ (2001) The mouse brain in stereotaxic coordinates. San Diego: Academic.

Rojas-Libano D, Frederick DE, Egaña JI, Kay LM (2014) The olfactory bulb theta rhythm follows all frequencies of diaphragmatic respiration in the freely behaving rat. Front Behav Neurosci 8:1-12. CrossRef Medline

Sainsbury RS, Montoya CP (1984) The relationship between type 2 theta and behavior. Physiol Behav 33:621-626. CrossRef Medline

Schomburg EW, Fernández-Ruiz A, Mizuseki K, Berényi A, Anastassiou CA, Koch C, Buzsáki G (2014) Theta phase segregation of input-specific gamma patterns in entorhinal-hippocampal networks. Neuron 84: 470-485. CrossRef Medline

Senut MC, Menetrey D, Lamour Y (1989) Cholinergic and peptidergic projections from the medial septum and the nucleus of the diagonal band of Broca to dorsal hippocampus, cingulate cortex and olfactory bulb: a combined wheat germ agglutinin-apo horseradish peroxidase-gold immunohistochemical study. Neuroscience 30:385-403. CrossRef Medline

Seth AK, Barrett AB, Barnett L (2015) Granger causality analysis in neuroscience and neuroimaging. J Neurosci 35:3293-3297. CrossRef Medline

Sirota A, Montgomery S, Fujisawa S, Isomura Y, Zugaro M, Buzsáki G (2008) Entrainment of neocortical neurons and gamma oscillations by the hippocampal theta rhythm. Neuron 60:683-697. CrossRef Medline

Smith JC, Ellenberger HH, Ballanyi K, Richter DW, Feldman JL (1991) PreBötzinger complex: a brainstem region that may generate respiratory rhythm in mammals. Science 254:726-729. CrossRef Medline

Sosulski DL, Bloom ML, Cutforth T, Axel R, Datta SR (2011) Distinct representations of olfactory information in different cortical centers. Nature 472:213-216. CrossRef Medline

Steriade M, Nuñez A, Amzica F (1993) A novel slow ( $<1 \mathrm{~Hz}$ ) oscillation of neocortical neurons in vivo: depolarizing and hyperpolarizing components. J Neurosci 13:3252-3265. Medline

Tsanov M, Chah E, Reilly R, O’Mara SM (2014) Respiratory cycle entrainment of septal neurons mediates the fast coupling of sniffing rate and hippocampal theta rhythm. Eur J Neurosci 39:957-974. CrossRef Medline

Vanderwolf CH (1969) Hippocampal electrical activity and voluntary movement in the rat. Electroencephalogr Clin Neurophysiol 26:407-418. CrossRef Medline 
Vanderwolf CH (1992) Hippocampal activity, olfaction, and sniffing: an olfactory input to the dentate gyrus. Brain Res 593:197-208. CrossRef Medline

Vanderwolf CH, Szechtman H (1987) Electrophysiological correlates of stereotyped sniffing in rats injected with apomorphine. Pharmacol Biochem Behav 26:299-304. CrossRef Medline

Viczko J, Sharma AV, Pagliardini S, Wolansky T, Dickson CT (2014) Lack of respiratory coupling with neocortical and hippocampal slow oscillations. J Neurosci 34:3937-3946. CrossRef Medline

Wilson RC, Steward O (1978) Polysynaptic activation of the dentate gyrus of the hippocampal formation: an olfactory input via the lateral entorhinal cortex. Exp Brain Res 33:523-534. Medline
Witter MP (1993) Organization of the entorhinal-hippocampal system: a review of current anatomical data. Hippocampus 3: 33-44. Medline

Wouterlood FG, Nederlof J (1983) Terminations of olfactory afferents on layer II and III neurons in the entorhinal area: degeneration-Golgielectron microscopic study in the rat. Neurosci Lett 36:105-110. CrossRef Medline

Xu W, Wilson DA (2012) Odor-evoked activity in the mouse lateral entorhinal cortex. Neuroscience 223:12-20. CrossRef Medline

Yanovsky Y, Ciatipis M, Draguhn A, Tort AB, Brankačk J (2014) Slow oscillations in the mouse hippocampus entrained by nasal respiration. J Neurosci 34:5949-5964. CrossRef Medline 